\title{
Non-planar Dynamics of a CFRP Cable with an External 1/3-order Subharmonic Resonance
}

\author{
Yang Long \\ Guangxi Polytechnic of Construction \\ Hou Jun Kang ( $\square$ houjun_kang@163.com ) \\ Guangxi University https://orcid.org/0000-0002-2452-9844
}

\section{Research Article}

Keywords: CFRP, Cable, Non-planar vibration, Internal resonance, Subharmonic resonance

Posted Date: November 9th, 2021

DOl: https://doi.org/10.21203/rs.3.rs-933386/v1

License: (c) (i) This work is licensed under a Creative Commons Attribution 4.0 International License. Read Full License 


\title{
Non-planar dynamics of a CFRP cable with an external 1/3-order subharmonic resonance
}

\author{
Yang Long Houjun Kang
}

\begin{abstract}
Based on the non-planar vibration equations of a cable made of carbon fiber reinforced polymer (CFRP), the nonlinear behaviors of the cable are studied. The one-to-one internal resonance of the lowest in-plane and out-of-plane modes of the cable is investigated. Three different cases, namely, 1/3-order subharmonic resonance of the in-plane mode, 1/3-order subharmonic resonance of the out-of-plane mode and 1/3-order subharmonic resonance of both of the in-plane and out-of-plane modes are examined. The vibration equations of the cable are discretized by using Galerkin's method. In this way, the ordinary differential equations (ODEs) are obtained. The multiple time scale method is employed to solve the ODEs and the corresponding modulation equations are derived. Then, the response curves of the cable are obtained by using Newton-Raphson method and pseudo arclength algorithm. Meanwhile, the response curves of the CFRP cable are compared with those of the steel cable to explore the differences in nonlinear behaviors of the cables made of different materials. The results show that the CFRP cable has potential advantages over the steel cable from a nonlinear point of view.
\end{abstract}

Keywords: CFRP; Cable; Non-planar vibration; Internal resonance; Subharmonic resonance

\section{Introduction}

Cables are extensively used in cable-supported system, such as cable-stayed [1] and suspension bridges [2]. Due to their low damping and small mass, the cables are prone to vibration under extreme environmental conditions (such as wind and rain). Therefore, nonlinear dynamics of the cables are always a hot topic in the field of civil engineering.

Irvine [3] first developed the linear dynamics of the cable and the linear modal analysis was carried out. Hagedorn and Schäfer [4] and Luongo et al. [5] studied the free vibration of an elastic cable. Benedettini and Rega [6-8] investigated the nonlinear dynamics of an elastic cable under planar excitation. By establishing a two-degree-of-freedom model, Perkins [9] explored modal interactions of elastic cables under parametric/external excitation. The results showed that a small support excitation could result in the parametric vibration of out-of-plane motion and simultaneous parametric and external vibrations of in-plane motion. Gattulli et al. [10] explored the interactions between planar and spatial modes subjected to in-plane and out-of-plane loads by utilizing analytical and finite element models. Zhao and Wang [11,12] considered a suspended cable and investigated three-to-one internal resonance between the symmetric modes by using discretization approach and direct approach, respectively. The rich nonlinear phenomena, such as torus bifurcation, period-doubling bifurcations, cyclic-fold bifurcation and chaotic motions were observed. The above studies present complex nonlinear behaviors and reveal the possibility of large vibration of the cable. However, these studies are mainly devoted to planar dynamics of the cable. Actually, one-to-one internal resonance between the in-plane and out-of-plane modes is possible for a cable $[13,14]$.

On the other hand, with the increase in the span of the cable-stayed bridge, the length and mass of the cable become larger and larger, which leads to reduce of the bearing efficiency of cable. Moreover, the deterioration of bridge durability due to corrosion of conventional steel cables is a major cause of bridge collapses and other devastating accidents. Recently, CFRP cable has been proposed to replace steel cable and it was used in practical engineering $[15,16]$. Quantities of researches on the CFRP cable have been conducted.

Su et al. [17] established a novel dynamic model with discrete springs for a floating cable-stayed bridge and solved the in-plane eigenvalue problem based upon transfer matrix method. The variation of the vertical stiffness of the bridge was explored when the steel cables were replaced by CFRP cables. Mei et al. [18] studied static and dynamic mechanical properties of a long-span cable-stayed bridge using CFRP cables. The results showed that the cable sag had a smaller effect on the CFRP cable

\footnotetext{
Y. Long

Department of Civil Engineering,Guangxi Polytechnic of Construction,Nanning 530007, China

H. Kang $(\bowtie)$

Scientific Research Center of Engineering Mechanics, Guangxi University, Nanning 530004, China

e-mail: houjun kang@163.com
} 
compared with traditional steel cable. The effects of the temperature on the steel cable were larger than those of the CFRP cable. Xie et al. [19] investigated the static and dynamic behaviors of cable-stayed bridges with CFRP cables from both experimental and numerical simulation perspectives. They pointed out that the mechanical behaviors of cable-stayed bridges using CFRP cables are significantly influenced by the nonlinearity. Moreover, the CFRP cables can improve the natural frequencies, especially the first-order frequency. Kang et al. [20] found that when the steel cable was replaced by the CFRP cable, the softening characteristic of the response curves would change to hardening one and the response amplitudes had an obvious decrease.

It can be summarized that the CFRP cable may exhibit different nonlinear characteristics from steel cable, because the CFRP has lighter weight and higher strength. In addition, the application of CFRP in practical engineering is still in the exploration stage, especially for large-span cable-stayed bridges. Hence, the nonlinear properties of the CFRP cable still need to be studied more deeply and broadly. More importantly, most of the studies are devoted to the internal resonance between in-plane and out-of-plane modes when primary resonance occurs. To the best of the authors' knowledge, the internal resonance between in-plane and out-of-plane modes under 1/3-order subharmonic resonance is seldom involved. Based on the above research background, this paper considers a CFRP cable and studies one-to-one internal resonance between the in-plane and out-of-plane modes when 1/3-order subharmonic resonance occurs. The response curves are given to explore the nonlinear behaviors of the CFRP cable. Meanwhile, the response curves of a steel cable are also presented and compared with those of the CFRP cable to reveal the differences in nonlinear behaviors of the cables made of different materials.

The remainder of the paper is organized as follows. First, the mechanical model and governing equations of the system are presented. In Section 3, the ODEs are solved by using multiple time scale method. Section 4 is devoted to numerical results and discussions. Finally, some interesting conclusions are drawn.

\section{Mechanical model and governing equations}

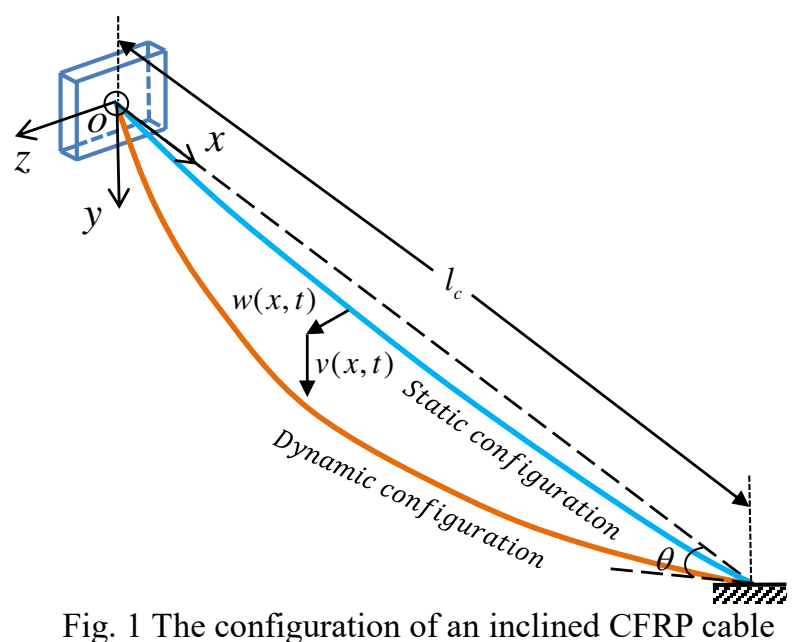

Fig. 1 describes the configurations of an inclined CFRP cable. The spatial Cartesian coordinate $x o y z$ is established to describe the motion of the cable. $v(x, t)$ and $w(x, t)$ denote in-plane and out-of-plane transverse displacements, respectively. The length of the cable is $l_{c}$. $\theta$ represents the acute angle between the cable and horizontal line. In order to explore the nonlinear dynamics of the CFRP cable subjected to wind load, the cable is subjected to two harmonic excitations (not drawn in the figure), namely, $p_{v}$ in the $y$-direction and $p_{w}$ in the $z$-direction. For the sake of derivation, the following assumptions are made:

(a) ignore the axial vibration of the CFRP cable;

(b) the problem we are concerned with is the nonlinear behaviors of the CFRP cable with clamped-clamped ends. Hence, it is suggested that the bending stiffness should be taken into account for the CFRP cable;

(c) the shear force of the CFRP cable is considered, since its shear rigidity is relatively smaller compared with that of a steel cable.

Based on the extended Hamilton's principle, the vibration equations governing the in-plane and out-of-plane motions of the cable are [21] 
$m \mu_{v} \& p_{v}-T_{0} v^{\prime \prime}-E A e(t)\left(y^{\prime \prime}+v^{\prime \prime}\right)+E I v^{\prime \prime \prime}+\frac{E I}{G A}\left[-m p_{v}-\mu_{v} \&+T_{0} v^{\prime \prime}+E A e(t)\left(y^{\prime \prime}+v^{\prime \prime}\right)\right]^{\prime \prime}=0(1)$

$$
m \mu_{w} \text { \&\& } p_{w}-T_{0} w^{\prime \prime}-\operatorname{EAe}(t) w^{\prime \prime}+\frac{E I}{G A}\left(-m p_{w}-\mu_{w} \iota \&+T_{0} w^{\prime \prime}+\operatorname{EAe}(t) w^{\prime \prime}+G A w^{\prime \prime}\right)^{\prime \prime}=0
$$

where $m, \mu, T_{0}, E, A, I$ and $G$ are mass per unit length, damping coefficient, initial force, Young's modulus, cross-sectional area, second moment of area and shear modulus, respectively. $E A, E I$ and $G A$ represent axial stiffness, bending stiffness and shear rigidity, respectively. The dot and prime denote the differentiations with respect to time $t$ and coordinate $x$, respectively. $e(t)$ denotes uniform dynamic elongation in the cable motion and can be expressed as

$$
e(t)=\frac{1}{l_{c}} \int_{0}^{l_{c}}\left(y^{\prime} v^{\prime}+\frac{1}{2} v^{\prime 2}+\frac{1}{2} w^{\prime 2}\right) \mathrm{d} x
$$

where $y$ denotes the static configuration of the cable. Following the reference [21], the expression of $y$ is

$$
y=\frac{b}{2 a}\left(x-x^{2}\right)-\frac{b}{2 a \sqrt{a}}\left(\operatorname{coth} \frac{\sqrt{a}}{2}+\operatorname{csch} \frac{\sqrt{a}}{2} \cosh \left(\frac{\sqrt{a}}{2}(1-2 x)\right)\right)
$$

where

$$
a=\frac{H l^{2}}{E I}, \quad b=\frac{m g l^{3}}{E I} \cos \theta
$$

To obtain the non-dimensional forms of the vibration equations, the following non-dimensional quantities are introduced

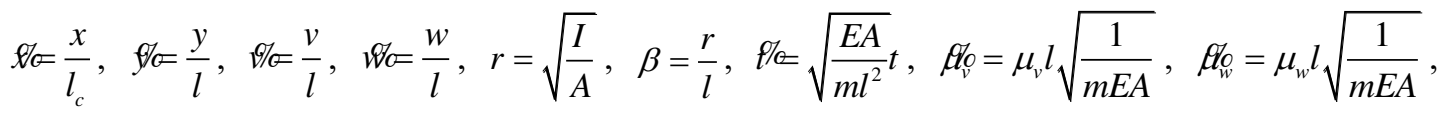

$$
\begin{aligned}
& \lambda=\frac{E A}{T_{0}}, \quad \xi=\frac{E}{G}, \quad p_{v}^{o}=\frac{p_{v} l}{E A}, \quad p_{w}^{o}=\frac{p_{w} l}{E A}
\end{aligned}
$$

In this way, Eqs. (1) (3) can be rewritten as the following equations by omitting the wave symbol

$$
\begin{gathered}
\mu_{v} \& \frac{1}{\lambda} v^{\prime \prime}-e\left(y^{\prime \prime}+v^{\prime \prime}\right)+\beta^{2} v^{\prime \prime \prime}-\xi \beta^{2}-\xi \beta^{2} \mu_{v} \mathbb{Q}^{\prime \prime}+\frac{\xi \beta^{2}}{\lambda} v^{\prime \prime \prime}+e \xi \beta^{2}\left(y^{\prime \prime \prime}+v^{\prime \prime \prime}\right)=p_{v} \\
\mu_{w} \text { w\& }-\frac{1}{\lambda} w^{\prime \prime}-e w^{\prime \prime}+\beta^{2} w^{\prime \prime}-\xi \beta^{2}-\xi \beta^{2} \mu_{w} \&^{\prime \prime}+\frac{\xi \beta^{2}}{\lambda} w^{\prime \prime \prime}+e \xi \beta^{2} w^{\prime \prime \prime}=p_{w} \\
e(t)=\int_{0}^{1}\left(y^{\prime} v^{\prime}+\frac{1}{2} v^{\prime 2}+\frac{1}{2} w^{\prime 2}\right) \mathrm{d} x
\end{gathered}
$$

The discrete systems can be derived by assuming $v(x, t)$ and $w(x, t)$ to be the following forms

$$
\begin{aligned}
& v(x, t)=\phi(x) g(t) \\
& w(x, t)=\varphi(x) q(t)
\end{aligned}
$$

where $g(t)$ and $q(t)$ are generalized coordinates of the lowest modes of in-plane and out-of-plane motion of the CFRP cable. $\phi(x)$ and $\varphi(x)$ are the corresponding shape functions of the CFRP cable and they can be taken as sine functions.

To obtain the ODEs of the system, Eqs. (8) and (9) are substituted into Eqs. (5) and (6). Then, the Galerkin's method is utilized. In this way, the following ODEs can be derived

$$
\begin{gathered}
\mu_{1} g+b_{11} g+b_{12} g^{2}+b_{13} q^{2}+b_{14} g^{3}+b_{15} g q^{2}+p_{1}=0 \\
\mu_{2} \&+b_{21} q+b_{22} g q+b_{23} g^{2} q+b_{24} q^{3}+p_{2}=0
\end{gathered}
$$

where $b_{11} \sim b_{15}$ and $b_{21} \sim b_{24}$ are Galerkin integral coefficients and they are reported in Appendix A.

In the following, the multiple time scale method is applied to solve the Eqs. (10) and (11). To this end, a small bookkeeping parameter $\varepsilon$ and new independent time variables $T_{0}=\varepsilon^{0} \tau$ and $T_{1}=\varepsilon^{1} \tau$ are introduced. Generally, $T_{0}$ is referred to as fast time scale and $T_{1}$ represents slow time scale. In order to explore the nonlinear dynamics of the CFRP cable when the external 1/3 order subharmonic resonance occurs, the three cases of external excitations are considered as follows, namely, in-plane excitation, out-of-plane excitation and both of the in-plane and out-of-plane excitations. 


\section{Multiple time scale method}

\subsection{In-plane excitation}

According to the basic principle of the multiple time scale method, the terms in Eqs. (10) and (11) are rescaled, such as the damping terms, exciting terms and nonlinear terms. Then, Eqs. (10) and (11) can be rewritten as

$$
\begin{gathered}
\omega_{1}^{2} g+\varepsilon \mu_{1} g+\varepsilon b_{12} g^{2}+\varepsilon b_{13} q^{2}+\varepsilon b_{14} g^{3}+\varepsilon b_{15} g q^{2}+f_{1} \cos \Omega_{1} t=0 \\
\omega_{2} q+\varepsilon \mu_{2} \&+\varepsilon b_{22} g q+\varepsilon b_{23} g^{2} q+\varepsilon b_{24} q^{3}=0
\end{gathered}
$$

where $\omega_{1}=\sqrt{b_{11}}$ and $\omega_{2}=\sqrt{b_{21}}$. They are in-plane natural frequency and out-of-plane natural frequency, respectively. Moreover, $f_{1} \cos \Omega_{1} t=p_{1}$.

To seek a first order approximation of the solutions, $g$ and $q$ are expressed as the power series of $\varepsilon$, namely

$$
\begin{aligned}
& g=g_{1}\left(T_{0}, T_{1}\right)+\varepsilon g_{2}\left(T_{0}, T_{1}\right)+O\left(\varepsilon^{2}\right) \\
& q=q_{1}\left(T_{0}, T_{1}\right)+\varepsilon q_{2}\left(T_{0}, T_{1}\right)+O\left(\varepsilon^{2}\right)
\end{aligned}
$$

Substituting Eqs. (14) and (15) into Eqs. (12) and (13) and equating terms of like order in $\varepsilon$ can derive the following expressions

order $\varepsilon^{0}$,

$$
\begin{aligned}
& \left(D_{0}^{2}+\omega_{1}^{2}\right) g_{1}+f_{1} \cos \Omega_{1} t=0 \\
& \left(D_{0}^{2}+\omega_{2}^{2}\right) q_{1}=0
\end{aligned}
$$

order $\varepsilon^{1}$,

$$
\begin{aligned}
& \left(D_{0}^{2}+\omega_{1}^{2}\right) g_{2}=-\left(b_{12} g_{1}^{2}+b_{13} q_{1}^{2}+b_{14} g_{1}^{3}+b_{15} g_{1} q_{1}^{2}+\mu_{1} D_{0}^{1} g_{1}+2 D_{1}^{1} g_{1}\right) \\
& \left(D_{0}^{2}+\omega_{2}^{2}\right) q_{2}=-\left(b_{22} g_{1} q_{1}+b_{23} g_{1}^{2} q_{1}+b_{24} q_{1}^{3}+\mu_{2} D_{0}^{1} q_{1}+2 D_{1}^{1} q_{1}\right)
\end{aligned}
$$

where $D_{n}^{m}(m=1,2$ and $n=0,1)$ is defined as $D_{n}^{m}=\frac{\partial^{m}}{\partial T_{n}}$. The solutions of the problem (43) are

$$
\begin{gathered}
g_{1}=A_{1}\left(T_{1}\right) \mathrm{e}^{\mathrm{i} \omega_{1} T}{ }_{0}+\frac{f_{1}}{2\left(\Omega_{1}^{2}-\omega_{1}^{2}\right)} \mathrm{e}^{\mathrm{i} \Omega_{1} T_{0}}+c c \\
q_{1}=A_{2}\left(T_{1}\right) \mathrm{e}^{\mathrm{i} \omega_{2} T_{0}}+c c
\end{gathered}
$$

where $A_{m}\left(T_{1}\right)$ is the complex amplitude and $c c$ denotes the complex conjugate of the preceding terms.

This paper is concerned with one-to-one internal resonance between the first in-plane and out-of-plane modes when 1/3-order subharmonic resonance occurs. Based on this, the following can be derived by substituting Eqs. (18) and (19) into Eq. (17), i.e.

$$
\begin{aligned}
\left(D_{0}^{2}+\omega_{1}^{2}\right) g_{2}= & \mathrm{i} \mu_{1} \omega_{1} A_{1} \mathrm{e}^{\mathrm{i} T_{0} \omega_{1}}+\frac{3 b_{14} f_{1}^{2} A_{1}}{2\left(\Omega_{1}^{2}-\omega_{1}^{2}\right)^{2}} \mathrm{e}^{\mathrm{i} T_{0} \omega_{1}}+3 b_{14} A_{1}^{2} B_{1} \mathrm{e}^{\mathrm{i} T_{0} \omega_{1}}+b_{15} A_{2}^{2} B_{1} \mathrm{e}^{2 \mathrm{i} T_{0} \omega_{2}-\mathrm{i} T_{0} \omega_{1}} \\
& +\frac{3 b_{14} f_{1} B_{1}^{2} \mathrm{e}^{\mathrm{i} T_{0} \Omega_{1}-2 \mathrm{i} T_{0} \omega_{1}}}{2\left(\Omega_{1}^{2}-\omega_{1}^{2}\right)}+2 b_{15} A_{1} A_{2} B_{2} \mathrm{e}^{\mathrm{i} T_{0} \omega_{1}}+\frac{b_{15} f_{1} B_{2}^{2}}{2\left(\Omega_{1}^{2}-\omega_{1}^{2}\right)} \mathrm{e}^{\mathrm{i} T_{0} \Omega_{1}-2 \mathrm{i} T_{0} \omega_{2}} \\
& +2 \mathrm{i} \omega_{1} \mathrm{e}^{\mathrm{i} T_{0} \omega_{1}} D_{1}^{1} A_{1}+N S T_{1}+c c \\
\left(D_{0}^{2}+\omega_{2}^{2}\right) q_{2}= & \mathrm{i} \mu_{2} \omega_{2} A_{2} \mathrm{e}^{\mathrm{i} T_{0} \omega_{2}}+\frac{b_{23} f_{1}^{2} A_{2}}{2\left(\Omega_{1}^{2}-\omega_{1}^{2}\right)^{2}} \mathrm{e}^{\mathrm{i} T_{0} \omega_{2}}+2 b_{23} A_{1} A_{2} B_{1} \mathrm{e}^{\mathrm{i} T_{0} \omega_{2}}+b_{23} A_{1}^{2} B_{2} \mathrm{e}^{2 \mathrm{i} T_{0} \omega_{1}-\mathrm{iT}_{0} \omega_{2}} \\
+ & 3 b_{24} A_{2}^{2} B_{2} \mathrm{e}^{\mathrm{i} T_{0} \omega_{2}}+\frac{b_{23} f_{1} B_{1} B_{2}}{\Omega_{1}^{2}-\omega_{1}^{2}} \mathrm{e}^{\mathrm{i} T_{0} \Omega_{1}-\mathrm{i} T_{0} \omega_{1}-\mathrm{i}_{0} \omega_{2}}+2 \mathrm{i} \omega_{2} \mathrm{e}^{\mathrm{i} T_{0} \omega_{2}} D_{1}^{1} A_{2}+N S T_{2}+c c
\end{aligned}
$$

where $B_{m}\left(T_{1}\right)$ is the complex conjugate of $A_{m}\left(T_{1}\right)$ and $N S T_{m}$ represent non-secular terms.

The nearness of the frequency relationships is described by introducing the external detuning parameters and internal detuning parameter, i.e.

$$
\Omega_{1}=3 \omega_{1}+\varepsilon \sigma \quad \omega_{2}=\omega_{1}+\varepsilon \sigma_{1}
$$

Meanwhile, to solve Eqs. (20) and (21), the polar transformations are adopted 


$$
A_{m}\left(T_{1}\right)=\frac{1}{2} a_{1}\left(T_{1}\right) \mathrm{e}^{\mathrm{i} \psi_{1}\left(T_{1}\right)} \quad B_{m}\left(T_{1}\right)=\frac{1}{2} a_{1}\left(T_{1}\right) \mathrm{e}^{-\mathrm{i} \psi_{1}\left(T_{1}\right)}
$$

Eq. (23) is substituted into Eqs. (20) and (21). Then, eliminating the secular terms and separating the real and imaginary parts respectively, the following autonomous modulation equations can be obtained

$$
\begin{gathered}
\omega_{1} \&=-\frac{1}{2} \mu_{1} \omega_{1} a_{1}-\frac{3 b_{14} f_{1} a_{1}^{2}}{8\left(\Omega_{1}^{2}-\omega_{1}^{2}\right)} \sin \alpha_{1}+\frac{b_{15} f_{1} a_{2}^{2}}{8\left(\Omega_{1}^{2}-\omega_{1}^{2}\right)} \sin \frac{1}{3}\left(2 \alpha_{2}-\alpha_{1}\right) \\
-\frac{1}{8} b_{15} a_{1} a_{2}^{2} \sin \frac{1}{3}\left(2 \alpha_{1}+2 \alpha_{2}\right) \\
\omega_{1} a_{1} \&=\omega_{1} a_{1} \sigma-\frac{9 b_{14} f_{1}^{2} a_{1}}{4\left(\Omega_{1}^{2}-\omega_{1}^{2}\right)^{2}}-\frac{9 b_{14} f_{1} a_{1}^{2}}{8\left(\Omega_{1}^{2}-\omega_{1}^{2}\right)} \cos \alpha_{1}-\frac{9}{8} b_{14} a_{1}^{3}-\frac{3 b_{15} f_{1} a_{2}^{2}}{8\left(\Omega_{1}^{2}-\omega_{1}^{2}\right)} \cos \frac{1}{3}\left(2 \alpha_{2}-\alpha_{1}\right) \\
-\frac{3}{4} b_{15} a_{1} a_{2}^{2}-\frac{3}{8} b_{15} a_{1} a_{2}^{2} \cos \frac{1}{3}\left(2 \alpha_{1}+2 \alpha_{2}\right) \\
\omega_{2} \&=-\frac{1}{2} \mu_{2} \omega_{2} a_{2}+\frac{b_{23} f_{1} a_{1} a_{2}}{4\left(\Omega_{1}^{2}-\omega_{1}^{2}\right)} \sin \frac{1}{3}\left(2 \alpha_{2}-\alpha_{1}\right)+\frac{1}{8} b_{23} a_{1}^{2} a_{2} \sin \frac{1}{3}\left(2 \alpha_{1}+2 \alpha_{2}\right) \\
\omega_{2} a_{2} \& \frac{3}{2}=3 \omega_{2} a_{2} \sigma_{1}-\omega_{2} a_{2} \sigma+\frac{3 b_{23} f_{1}^{2} a_{2}}{4\left(\Omega_{1}^{2}-\omega_{1}^{2}\right)^{2}}+\frac{3 b_{23} f_{1} a_{1} a_{2}}{4\left(\Omega_{1}^{2}-\omega_{1}^{2}\right)} \cos \frac{1}{3}\left(2 \alpha_{2}-\alpha_{1}\right)+\frac{3}{4} b_{23} a_{1}^{2} a_{2} \\
+\frac{3}{8} b_{23} a_{1}^{2} a_{2} \cos \frac{1}{3}\left(2 \alpha_{1}+2 \alpha_{2}\right)+\frac{9}{8} b_{24} a_{2}^{3}
\end{gathered}
$$

where $\alpha_{1}=T_{1} \sigma-3 \psi_{1}$ and $\alpha_{2}=3 T_{1} \sigma_{1}-T_{1} \sigma+3 \psi_{2}$. Letting $\&=\alpha=\&=\alpha=0$, the steady state solutions of the system can be obtained by Newton-Raphson method and pseudo arclength algorithm [22]. The stability of the equilibrium solution can be determined by checking whether the real part of the eigenvalue corresponding to linear matrix of the system is negative or not [23].

3.2. Out-of-plane excitation

In this part, the nonlinear solutions of the CFRP cable subjected to only out-of-plane excitation is given. Similarly, Eqs. (10) and (11) are rewritten as

$$
\begin{gathered}
\omega_{1}^{2} g+\varepsilon \mu_{1} \&+\varepsilon b_{12} g^{2}+\varepsilon b_{13} q^{2}+\varepsilon b_{14} g^{3}+\varepsilon b_{15} g q^{2}=0 \\
\omega_{2} q+\varepsilon \mu_{2} \&+\varepsilon b_{22} g q+\varepsilon b_{23} g^{2} q+\varepsilon b_{24} q^{3}+f_{2} \cos \Omega_{2} t=0
\end{gathered}
$$

where $f_{2} \cos \Omega_{1} t=p_{2}$.

Substituting Eqs. (14) and (15) into Eqs. (28) and (29) and equating terms of like order in $\varepsilon$ yields order $\varepsilon^{0}$,

$$
\begin{aligned}
& \left(D_{0}^{2}+\omega_{1}^{2}\right) g_{1}=0 \\
& \left(D_{0}^{2}+\omega_{2}^{2}\right) q_{1}+f_{2} \cos \Omega_{2} t=0
\end{aligned}
$$

order $\varepsilon^{1}$,

$$
\begin{aligned}
& \left(D_{0}^{2}+\omega_{1}^{2}\right) g_{2}=-\left(b_{12} g_{1}^{2}+b_{13} q_{1}^{2}+b_{14} g_{1}^{3}+b_{15} g_{1} q_{1}^{2}+\mu_{1} D_{0}^{1} g_{1}+2 D_{1}^{1} g_{1}\right) \\
& \left(D_{0}^{2}+\omega_{2}^{2}\right) q_{2}=-\left(b_{22} g_{1} q_{1}+b_{23} g_{1}^{2} q_{1}+b_{24} q_{1}^{3}+\mu_{2} D_{0}^{1} q_{1}+2 D_{1}^{1} q_{1}\right)
\end{aligned}
$$

The solutions of the problem (30) are

$$
\begin{gathered}
g_{1}=A_{1}\left(T_{1}\right) \mathrm{e}^{\mathrm{i} \omega_{1} T_{0}}+c c \\
q_{1}=A_{2}\left(T_{1}\right) \mathrm{e}^{\mathrm{i} \omega_{2} T_{0}}+\frac{f_{2}}{2\left(\Omega_{2}^{2}-\omega_{2}^{2}\right)} \mathrm{e}^{\mathrm{i} \Omega_{2} T_{0}}+c c
\end{gathered}
$$

Substituting Eqs. (32) and (33) into Eq. (31) leads to

$$
\begin{aligned}
\left(D_{0}^{2}+\omega_{1}^{2}\right) g_{2} & =\mathrm{i} \mu_{1} \omega_{1} A_{1} \mathrm{e}^{\mathrm{i} T_{0} \omega_{1}}+\frac{b_{15} f_{2}^{2} A_{1}}{2\left(\Omega_{2}^{2}-\omega_{2}^{2}\right)^{2}} \mathrm{e}^{\mathrm{i} T_{0} \omega_{1}}+3 b_{14} A_{1}^{2} B_{1} \mathrm{e}^{\mathrm{i} T_{0} \omega_{1}}+b_{15} A_{2}^{2} B_{1} \mathrm{e}^{2 \mathrm{i} T_{0} \omega_{2}-\mathrm{i} T_{0} \omega_{1}} \\
& +2 b_{15} A_{1} A_{2} B_{2} \mathrm{e}^{\mathrm{i} T_{0} \omega_{1}}+\frac{b_{15} f_{2} B_{1} B_{2}}{\Omega_{2}^{2}-\omega_{2}^{2}} \mathrm{e}^{\mathrm{i} T_{0} \Omega_{2}-\mathrm{i} T_{0} \omega_{1}-\mathrm{i} T_{0} \omega_{2}}+2 \mathrm{i} \omega_{1} \mathrm{e}^{\mathrm{i} T_{0} \omega_{1}} D_{1}^{1} A_{1}+N S T_{3}+c c
\end{aligned}
$$




$$
\begin{aligned}
\left(D_{0}^{2}+\omega_{2}^{2}\right) q_{2} & =\mathrm{i} \mu_{2} \omega_{2} A_{2} \mathrm{e}^{\mathrm{i} T_{0} \omega_{2}}+\frac{3 b_{24} f_{2}^{2} A_{2}}{2\left(\Omega_{2}^{2}-\omega_{2}^{2}\right)^{2}} \mathrm{e}^{\mathrm{i} T_{0} \omega_{2}}+2 b_{23} A_{1} A_{2} B_{1} \mathrm{e}^{\mathrm{i} T_{0} \omega_{2}}+\frac{b_{23} f_{2} B_{1}^{2}}{2\left(\Omega_{2}^{2}-\omega_{2}^{2}\right)} \mathrm{e}^{\mathrm{i} T_{0} \Omega_{2}-2 \mathrm{i} T_{0} \omega_{1}} \\
& +b_{23} A_{1}^{2} B_{2} \mathrm{e}^{2 \mathrm{i} T_{0} \omega_{1}-\mathrm{i} T_{0} \omega_{2}}+3 b_{24} A_{2}^{2} B_{2} \mathrm{e}^{\mathrm{i} T_{0} \omega_{2}}+\frac{3 b_{24} f_{2} B_{2}^{2}}{2\left(\Omega_{2}^{2}-\omega_{2}^{2}\right)} \mathrm{e}^{\mathrm{i} T_{0} \Omega_{2}-2 \mathrm{i} T_{0} \omega_{2}}+2 \mathrm{i} \omega_{2} \mathrm{e}^{\mathrm{i} T_{0} \omega_{2}} D_{1}^{1} A_{2} \\
& +N S T_{4}+c c
\end{aligned}
$$

where $N S T_{3}$ and $N S T_{4}$ represent non-secular terms.

The nearness of the frequency relationships is described by introducing the external detuning parameters and internal detuning parameter, i.e.

$$
\Omega_{2}=3 \omega_{1}+\varepsilon \sigma \quad \omega_{2}=\omega_{1}+\varepsilon \sigma_{1}
$$

Meanwhile, Eq. (23) is substituted into Eqs. (34) and (35). Using the same processing as in the previous section, the autonomous modulation equations corresponding to out-of-plane excitation can be obtained, namely

$$
\begin{aligned}
\omega_{1} \&= & -\frac{1}{2} \mu_{1} \omega_{1} a_{1}+\frac{b_{15} f_{2} a_{1} a_{2}}{4\left(\Omega_{2}^{2}-\omega_{2}^{2}\right)} \sin \frac{1}{3}\left(\alpha_{2}-2 \alpha_{1}\right)-\frac{1}{8} b_{15} a_{1} a_{2}^{2} \sin \frac{1}{3}\left(2 \alpha_{1}+2 \alpha_{2}\right) \\
\omega_{1} a_{1} \& \&= & \omega_{1} a_{1} \sigma-\frac{3 b_{15} f_{2}^{2} a_{1}}{4\left(\Omega_{2}^{2}-\omega_{2}^{2}\right)^{2}}-\frac{9}{8} b_{14} a_{1}^{3}-\frac{3 b_{15} f_{2} a_{2} a_{1}}{4\left(\Omega_{2}^{2}-\omega_{2}^{2}\right)} \cos \frac{1}{3}\left(\alpha_{2}-2 \alpha_{1}\right)-\frac{3}{4} b_{15} a_{1} a_{2}^{2} \\
& -\frac{3}{8} b_{15} a_{1} a_{2}^{2} \cos \frac{1}{3}\left(2 \alpha_{1}+2 \alpha_{2}\right) \\
\omega_{2} \&= & -\frac{1}{2} \mu_{2} \omega_{2} a_{2}+\frac{b_{23} f_{2} a_{1}^{2}}{8\left(\Omega_{2}^{2}-\omega_{2}^{2}\right)} \sin \frac{1}{3}\left(\alpha_{2}-2 \alpha_{1}\right)+\frac{1}{8} b_{23} a_{1}^{2} a_{2} \sin \frac{1}{3}\left(2 \alpha_{1}+2 \alpha_{2}\right) \\
+ & \frac{3 b_{24} f_{2} a_{2}^{2}}{8\left(\Omega_{2}^{2}-\omega_{2}^{2}\right)} \sin \alpha_{2} \\
\omega_{2} a_{2} \alpha_{2}= & 3 \omega_{2} a_{2} \sigma_{1}-\omega_{2} a_{2} \sigma+\frac{9 b_{24} f_{2}^{2} a_{2}}{4\left(\Omega_{2}^{2}-\omega_{2}^{2}\right)^{2}}+\frac{3}{4} b_{23} a_{1}^{2} a_{2}+\frac{3}{8} b_{23} a_{1}^{2} a_{2} \cos \frac{1}{3}\left(2 \alpha_{1}+2 \alpha_{2}\right) \\
& +\frac{3 b_{23} f_{2} a_{1}^{2}}{8\left(\Omega_{2}^{2}-\omega_{2}^{2}\right)} \cos \frac{1}{3}\left(\alpha_{2}-2 \alpha_{1}\right)+\frac{9 b_{24} f_{2} a_{2}^{2}}{8\left(\Omega_{2}^{2}-\omega_{2}^{2}\right)} \cos \alpha_{2}+\frac{9}{8} b_{24} a_{2}^{3}
\end{aligned}
$$

3.3. Exciting both of the in-plane and out-of-plane modes

For the case that the CFRP cable is subjected to both of the in-plane and out-of-plane excitations, Eqs. (10) and (11) can be rewritten as

$$
\begin{gathered}
\omega_{1}^{2} g+\varepsilon \mu_{1} \&+\varepsilon b_{12} g^{2}+\varepsilon b_{13} q^{2}+\varepsilon b_{14} g^{3}+\varepsilon b_{15} g q^{2}+f_{1} \cos \Omega_{1} t=0 \\
\omega_{2} q+\varepsilon \mu_{2} \&+\varepsilon b_{22} g q+\varepsilon b_{23} g^{2} q+\varepsilon b_{24} q^{3}+f_{2} \cos \Omega_{2} t=0
\end{gathered}
$$

Substituting Eqs. (14) and (15) into Eqs. (41) and (42) and equating terms of like order in $\varepsilon$ can derive the following equations order $\varepsilon^{0}$,

$$
\begin{aligned}
& \left(D_{0}^{2}+\omega_{1}^{2}\right) g_{1}+f_{1} \cos \Omega_{1} t=0 \\
& \left(D_{0}^{2}+\omega_{2}^{2}\right) q_{1}+f_{2} \cos \Omega_{2} t=0
\end{aligned}
$$

order $\varepsilon^{1}$

$$
\begin{aligned}
& \left(D_{0}^{2}+\omega_{1}^{2}\right) g_{2}=-\left(b_{12} g_{1}^{2}+b_{13} q_{1}^{2}+b_{14} g_{1}^{3}+b_{15} g_{1} q_{1}^{2}+\mu_{1} D_{0}^{1} g_{1}+2 D_{1}^{1} g_{1}\right) \\
& \left(D_{0}^{2}+\omega_{2}^{2}\right) q_{2}=-\left(b_{22} g_{1} q_{1}+b_{23} g_{1}^{2} q_{1}+b_{24} q_{1}^{3}+\mu_{2} D_{0}^{1} q_{1}+2 D_{1}^{1} q_{1}\right)
\end{aligned}
$$

The solutions of the problem (43) are

$$
\begin{aligned}
& g_{1}=A_{1}\left(T_{1}\right) \mathrm{e}^{\mathrm{i} \omega_{1} T_{0}}+\frac{f_{1}}{2\left(\Omega_{1}^{2}-\omega_{1}^{2}\right)} \mathrm{e}^{\mathrm{i} \Omega_{1} T_{0}}+c c \\
& q_{1}=A_{2}\left(T_{1}\right) \mathrm{e}^{\mathrm{i} \omega_{2} T_{0}}+\frac{f_{2}}{2\left(\Omega_{2}^{2}-\omega_{2}^{2}\right)} \mathrm{e}^{\mathrm{i} \Omega_{2} T_{0}}+c c
\end{aligned}
$$

For the sake of analysis, a factor $k$ is introduced to describe relative magnitude of the two excitations, i.e.

$$
f_{2}=k f_{1}
$$


Substituting Eqs. (45) and (46) into Eq. (44) leads to

$$
\begin{aligned}
& \left(D_{0}^{2}+\omega_{1}^{2}\right) g_{2}=\mathrm{i} \mu_{1} \omega_{1} A_{1} \mathrm{e}^{\mathrm{i} T_{0} \omega_{1}}+\frac{3 b_{14} f_{1}^{2} A_{1} \mathrm{e}^{\mathrm{i} T_{0} \omega_{1}}}{2\left(\Omega_{1}^{2}-\omega_{1}^{2}\right)^{2}}+\frac{k^{2} b_{15} f_{1}^{2} A_{1} \mathrm{e}^{\mathrm{i} T_{0} \omega_{1}}}{2\left(\Omega_{2}^{2}-\omega_{2}^{2}\right)^{2}}+\frac{k b_{15} f_{1}^{2} A_{2} \mathrm{e}^{\mathrm{i} T_{0} \omega_{2}+\mathrm{i} T_{0} \Omega_{1}-\mathrm{i} T_{0} \Omega_{2}}}{2\left(\Omega_{1}^{2}-\omega_{1}^{2}\right)\left(\Omega_{2}^{2}-\omega_{2}^{2}\right)} \\
& +\frac{k b_{15} f_{1}^{2} A_{2} \mathrm{e}^{\mathrm{i} T_{0} \omega_{2}-\mathrm{i} T_{0} \Omega_{1}+\mathrm{i} T_{0} \Omega_{2}}}{2\left(\Omega_{1}^{2}-\omega_{1}^{2}\right)\left(\Omega_{2}^{2}-\omega_{2}^{2}\right)}+3 b_{14} A_{1}^{2} B_{1} \mathrm{e}^{\mathrm{i} T_{0} \omega_{1}}+b_{15} A_{2}^{2} B_{1} \mathrm{e}^{2 \mathrm{iT}_{0} \omega_{2}-\mathrm{iT}_{0} \omega_{1}} \\
& +\frac{3 b_{14} f_{1} B_{1}^{2} \mathrm{e}^{\mathrm{i} T_{0} \Omega_{1}-2 \mathrm{i} T_{0} \omega_{1}}}{2\left(\Omega_{1}^{2}-\omega_{1}^{2}\right)}+2 b_{15} A_{1} A_{2} B_{2} \mathrm{e}^{\mathrm{i} T_{0} \omega_{1}}+\frac{k b_{15} f_{1} B_{1} B_{2} \mathrm{e}^{\mathrm{i} T_{0} \Omega_{2}-\mathrm{i} T_{0} \omega_{1}-\mathrm{i} T_{0} \omega_{2}}}{\Omega_{2}^{2}-\omega_{2}^{2}} \\
& +\frac{b_{15} f_{1} B_{2}^{2} \mathrm{e}^{\mathrm{i} T_{0} \Omega_{1}-2 \mathrm{i} T_{0} \omega_{2}}}{2\left(\Omega_{1}^{2}-\omega_{1}^{2}\right)}+2 \mathrm{i} \omega_{1} \mathrm{e}^{\mathrm{i} T_{0} \omega_{1}} D_{1}^{1} A_{1}+N S T_{5}+c c \\
& \left(D_{0}^{2}+\omega_{2}^{2}\right) q_{2}=\frac{k b_{23} f_{1}^{2} A_{1} \mathrm{e}^{\mathrm{i} T_{0} \omega_{1}+\mathrm{i}_{0} \Omega_{1}-\mathrm{i} T_{0} \Omega_{2}}}{2\left(\Omega_{1}^{2}-\omega_{1}^{2}\right)\left(\Omega_{2}^{2}-\omega_{2}^{2}\right)}+\frac{k b_{23} f_{1}^{2} A_{1} \mathrm{e}^{\mathrm{i} \mathrm{T}_{0} \omega_{1}-\mathrm{i} T_{0} \Omega_{1}+\mathrm{i} T_{0} \Omega_{2}}}{2\left(\Omega_{1}^{2}-\omega_{1}^{2}\right)\left(\Omega_{2}^{2}-\omega_{2}^{2}\right)}+\mathrm{i} \mu_{2} \omega_{2} A_{2} \mathrm{e}^{\mathrm{i} T_{0} \omega_{2}} \\
& +\frac{b_{23} f_{1}^{2} A_{2} \mathrm{e}^{\mathrm{i} T_{0} \omega_{2}}}{2\left(\Omega_{1}^{2}-\omega_{1}^{2}\right)^{2}}+\frac{3 k^{2} b_{24} f_{1}^{2} A_{2} \mathrm{e}^{\mathrm{i} T_{0} \omega_{2}}}{2\left(\Omega_{2}^{2}-\omega_{2}^{2}\right)^{2}}+2 b_{23} A_{1} A_{2} B_{1} \mathrm{e}^{\mathrm{i} T_{0} \omega_{2}}+\frac{k b_{23} f_{1} B_{1}^{2} \mathrm{e}^{\mathrm{i} T_{0} \Omega_{2}-2 i T_{0} \omega_{1}}}{2\left(\Omega_{2}^{2}-\omega_{2}^{2}\right)} \\
& +b_{23} A_{1}^{2} B_{2} \mathrm{e}^{2 \mathrm{i}_{0} \omega_{1}-\mathrm{iT}_{0} \omega_{2}}+3 b_{24} A_{2}^{2} B_{2} \mathrm{e}^{\mathrm{i} T_{0} \omega_{2}}+\frac{b_{23} f_{1} B_{1} B_{2} \mathrm{e}^{\mathrm{i} T_{0} \Omega_{1}-\mathrm{i} T_{0} \omega_{1}-\mathrm{i} \mathrm{T}_{0} \omega_{2}}}{\Omega_{1}^{2}-\omega_{1}^{2}} \\
& +\frac{3 k b_{24} f_{1} B_{2}^{2} \mathrm{e}^{\mathrm{i} T_{0} \Omega_{2}-2 \mathrm{i}_{0} \omega_{2}}}{2\left(\Omega_{2}^{2}-\omega_{2}^{2}\right)}+2 \mathrm{i} \omega_{2} D_{1}^{1} A_{2} \mathrm{e}^{\mathrm{i} T_{0} \omega_{2}}+N S T_{6}+c c
\end{aligned}
$$

where $N S T_{5}$ and $N S T_{6}$ represent non-secular terms.

Here, the two excitation frequencies are assumed to be identical, namely

$$
\Omega_{1}=\Omega_{2}=3 \omega_{1}+\varepsilon \sigma \quad \omega_{2}=\omega_{1}+\varepsilon \sigma_{1}
$$

Substituting Eq. (23) into Eqs. (48) and (49) and considering Eq. (50), the autonomous modulation equations can be derived, namely

$$
\begin{aligned}
& \omega_{1} \&=-\frac{1}{2} \mu_{1} \omega_{1} a_{1}-\frac{3 b_{14} f_{1} a_{1}^{2} \sin \alpha_{1}}{8\left(\Omega_{1}^{2}-\omega_{1}^{2}\right)}-\frac{k b_{15} f_{1}^{2} a_{2}}{2\left(\Omega_{1}^{2}-\omega_{1}^{2}\right)\left(\Omega_{1}^{2}-\omega_{2}^{2}\right)} \sin \frac{1}{6}\left(2 \alpha_{1}+2 \alpha_{2}\right) \\
& +\frac{k b_{15} f_{1} a_{1} a_{2}}{4\left(\Omega_{1}^{2}-\omega_{2}^{2}\right)} \sin \frac{1}{3}\left(\alpha_{2}-2 \alpha_{1}\right)+\frac{b_{15} f_{1} a_{2}^{2} \sin \frac{1}{3}\left(2 \alpha_{2}-\alpha_{1}\right)}{8\left(\Omega_{1}^{2}-\omega_{1}^{2}\right)}-\frac{1}{8} b_{15} a_{1} a_{2}^{2} \sin \frac{1}{3}\left(2 \alpha_{1}+2 \alpha_{2}\right) \\
& \omega_{1} a_{1} \& \&=\omega_{1} a_{1} \sigma-\frac{9 b_{14} f_{1}^{2} a_{1}}{4\left(\Omega_{1}^{2}-\omega_{1}^{2}\right)^{2}}-\frac{3 k^{2} b_{15} f_{1}^{2} a_{1}}{4\left(\Omega_{1}^{2}-\omega_{2}^{2}\right)^{2}}-\frac{9 b_{14} f_{1} a_{1}^{2}}{8\left(\Omega_{1}^{2}-\omega_{1}^{2}\right)} \cos \alpha_{1}-\frac{9}{8} b_{14} a_{1}^{3} \\
& -\frac{3 k b_{15} f_{1}^{2} a_{2}}{2\left(\Omega_{1}^{2}-\omega_{1}^{2}\right)\left(\Omega_{1}^{2}-\omega_{2}^{2}\right)} \cos \frac{1}{6}\left(2 \alpha_{1}+2 \alpha_{2}\right)-\frac{3 k b_{15} f_{1} a_{1} a_{2}}{4\left(\Omega_{1}^{2}-\omega_{2}^{2}\right)} \cos \frac{1}{3}\left(\alpha_{2}-2 \alpha_{1}\right) \\
& -\frac{3 b_{15} f_{1} a_{2}^{2}}{8\left(\Omega_{1}^{2}-\omega_{1}^{2}\right)} \cos \frac{1}{3}\left(2 \alpha_{2}-\alpha_{1}\right)-\frac{3}{4} b_{15} a_{1} a_{2}^{2}-\frac{3}{8} b_{15} a_{1} a_{2}^{2} \cos \frac{1}{3}\left(2 \alpha_{1}+2 \alpha_{2}\right) \\
& \omega_{2} \alpha_{2}=-\frac{1}{2} \mu_{2} \omega_{2} a_{2}+\frac{k b_{23} f_{1}^{2} a_{1}}{2\left(\Omega_{1}^{2}-\omega_{1}^{2}\right)\left(\Omega_{1}^{2}-\omega_{2}^{2}\right)} \sin \frac{1}{6}\left(2 \alpha_{1}+2 \alpha_{2}\right)+\frac{k b_{23} f_{1} a_{1}^{2}}{8\left(\Omega_{1}^{2}-\omega_{2}^{2}\right)} \sin \frac{1}{3}\left(\alpha_{2}-2 \alpha_{1}\right) \\
& +\frac{b_{23} f_{1} a_{1} a_{2}}{4\left(\Omega_{1}^{2}-\omega_{1}^{2}\right)} \sin \frac{1}{3}\left(2 \alpha_{2}-\alpha_{1}\right)+\frac{1}{8} b_{23} a_{1}^{2} a_{2} \sin \frac{1}{3}\left(2 \alpha_{1}+2 \alpha_{2}\right)+\frac{3 k b_{24} f_{1} a_{2}^{2}}{8\left(\Omega_{1}^{2}-\omega_{2}^{2}\right)} \sin \alpha_{2} \\
& \omega_{2} a_{2} \alpha \frac{\alpha}{2}=3 \omega_{2} a_{2} \sigma_{1}-\omega_{2} a_{2} \sigma+\frac{3 k b_{23} f_{1}^{2} a_{1}}{2\left(\Omega_{1}^{2}-\omega_{1}^{2}\right)\left(\Omega_{1}^{2}-\omega_{2}^{2}\right)} \cos \frac{1}{6}\left(2 \alpha_{1}+2 \alpha_{2}\right) \\
& +\frac{3 k b_{23} f_{1} a_{1}^{2}}{8\left(\Omega_{1}^{2}-\omega_{2}^{2}\right)} \cos \frac{1}{3}\left(\alpha_{2}-2 \alpha_{1}\right)+\frac{3 b_{23} f_{1}^{2} a_{2}}{4\left(\Omega_{1}^{2}-\omega_{1}^{2}\right)^{2}}+\frac{9 k^{2} b_{24} f_{1}^{2} a_{2}}{4\left(\Omega_{1}^{2}-\omega_{2}^{2}\right)^{2}} \\
& +\frac{3 b_{23} f_{1} a_{1} a_{2}}{4\left(\Omega_{1}^{2}-\omega_{1}^{2}\right)} \cos \frac{1}{3}\left(2 \alpha_{2}-\alpha_{1}\right)+\frac{3}{4} b_{23} a_{1}^{2} a_{2}+\frac{3}{8} b_{23} a_{1}^{2} a_{2} \cos \frac{1}{3}\left(2 \alpha_{1}+2 \alpha_{2}\right) \\
& +\frac{9 k b_{24} f_{1} a_{2}^{2}}{8\left(\Omega_{1}^{2}-\omega_{2}^{2}\right)} \cos \alpha_{2}+\frac{9}{8} b_{24} a_{2}^{3}
\end{aligned}
$$




\section{Numerical results and discussions}

This section will discuss the nonlinear behaviors of the CFRP cable subjected to the external $1 / 3$ order subharmonic resonance. To explore the effects of different materials on the nonlinear behaviors of the cable, a steel cable is also chosen to be an example. The relevant parameters of these two cables are presented in Table 1. In the following analysis, SN and HB denote saddle-node and Hopf bifurcations, respectively. Stable solutions are depicted by thick lines, while unstable solutions are described by thin lines.

Table 1 Physical parameters of the three cables made of different materials

\begin{tabular}{cccccccccc}
\hline cables & $L / \mathrm{m}$ & $E /(\mathrm{GPa})$ & $A / \mathrm{m}^{2}$ & $m /(\mathrm{kg} / \mathrm{m})$ & $\theta / \mathrm{rad}$ & $T_{0} /(\mathrm{N})$ & $G(\mathrm{GPa})$ & $\mu_{1}$ & $\mu_{2}$ \\
\hline CFRP & 500 & 600 & 0.01719 & 20.92 & 0.393 & $6 \times 10^{6}$ & 3.75 & 0.003 & 0.003 \\
steel & 500 & 195 & 0.01719 & 99.92 & 0.393 & $6 \times 10^{6}$ & 3.75 & 0.003 & 0.003 \\
\hline
\end{tabular}

\subsection{In-plane excitation}

Fig. 2 shows the frequency-response curves of the CFRP cable when the external 1/3-order subharmonic resonance of in-plane mode occurs. Here, the excitation amplitude is chosen to be 0.01 . It is firstly noted that the response amplitudes of both in-plane and out-of-plane is smaller than results in the reference [21] when the external primary resonance is excited. It is also seen from Fig. 2 that the cable exhibits relatively complex nonlinear behaviors. There are stable solutions only in a certain range of excitation frequency. When excitation frequency (i.e., $\sigma$ ) increases from a relatively small value, two branches emerge at SN1. One branch is stable, while another branch is unstable. The unstable one is increased with the increase in excitation frequency and end at $\sigma=0.66$. The stable one also increases with the increase in excitation frequency and loses its stability at the point SN2. After that, the unstable solutions are larger than stable solutions at the beginning and then decrease with the increase in excitation frequency.

Nevertheless, the out-of-plane amplitude exhibits different behaviors. For example, as the excitation frequency is increased, the unstable solutions generated from SN1 increase firstly and then decrease to zero sharply. After SN2, the unstable solutions are smaller than stable solutions, while the subsequent increase makes the unstable solutions gradually exceed stable solutions with the increase in excitation frequency. Comparing the two figures in Fig. 2, it can be found that the out-of-plane amplitude is a little larger than in-plane one even though the cable is subjected to an in-plane excitation. In addition, the response curves in Fig. 2 do not exhibit softening or hardening characteristics, which is different from those when the primary resonance occurs.
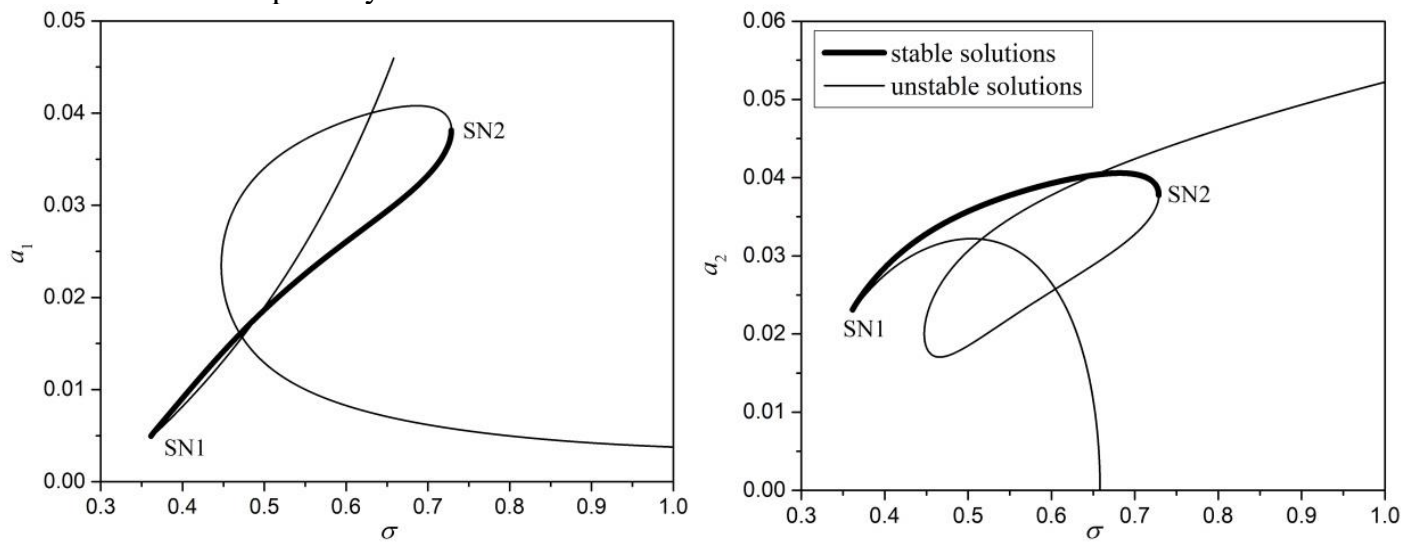

Fig. 2 The frequency-response curves of the CFRP cable with excitation amplitude $f_{1}=0.01$ and $\sigma_{1}=-$ 0.0013 .

In order to explore the effect of excitation amplitudes on the nonlinear behaviors of the CFRP cable, Fig. 3 is given. It can be seen that when the excitation amplitude is bigger, the solutions become relatively simple and there are only two branches in each paragraph. One is stable and the other one is unstable (see $f_{1}=0.02$ and $f_{1}=0.03$ ). The number of saddle-node bifurcation point decreases and there is only one SN bifurcation left for a bigger excitation amplitude. The above phenomena indicate that nonlinear behaviors of the CFRP cable will become simpler with the increase in excitation amplitude. This is similar to the results in the reference [21] when the external primary resonance is excited. In addition, when $f_{1}=0.02$ and $f_{1}=0.03$, the range of stable solutions expands compared with $f_{1}=0.01$. Observing Fig. 3, an interesting phenomenon can be found. When the excitation amplitude is increased 
to $0.03, a_{1}$ becomes smaller compared with $f_{1}=0.01$, but $a_{2}$ increases. This indicates that more energy is transferred into out-of-plane vibration through internal resonance.
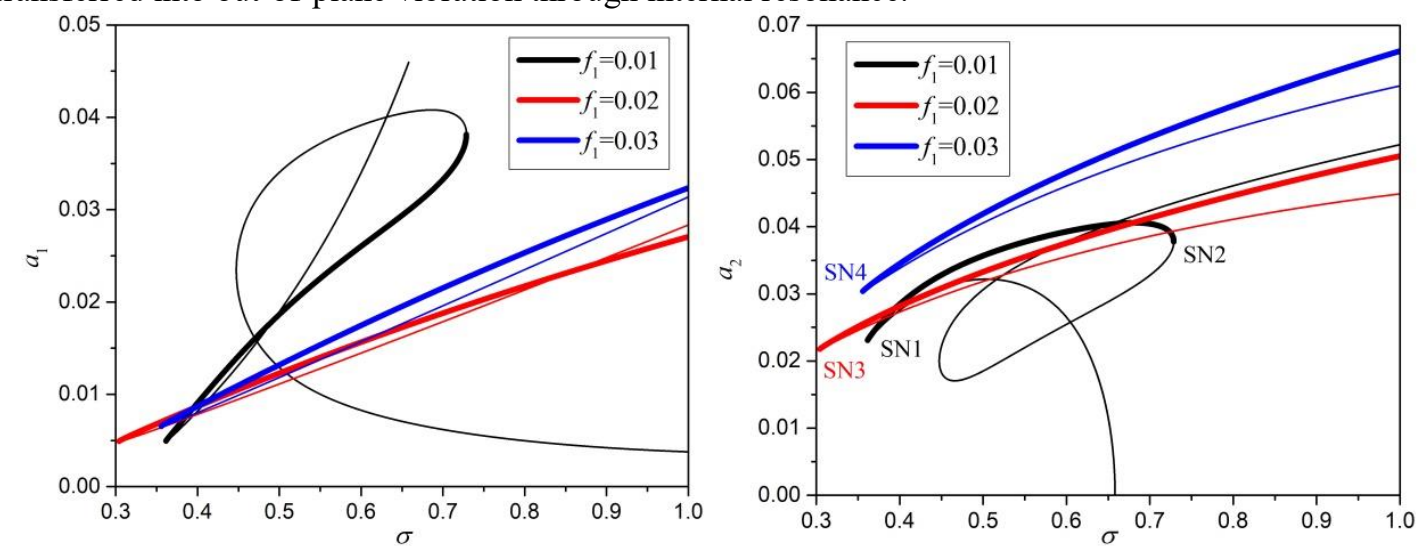

Fig. 3 The frequency-response curves of the CFRP cable with different excitation amplitudes when $\sigma_{1}=$ -0.0013 .

Fig. 4 presents the force-response curves of the CFRP cable when external detuning parameter $\sigma=0.6$. Similar to the frequency-response curves, there are solutions only when the excitation amplitude is in a certain range. When the excitation amplitude increases from a relatively small value, the unstable solutions decreased to the bottom sharply from a large value. This means that under specific initial conditions, large unstable vibrations may occur in the cable's vibration, which should attract our attention in practical engineering. When the excitation force increases, the SN1 occurs, through which the response gains its stability. Then, the response amplitude decreases with the increase in excitation amplitude until SN2 is reached, via which the response regains the stability. From then on, the response amplitude increases 'inversely' with the variation of excitation amplitude.

However, $a_{2}$ exhibits almost the opposite nonlinear behaviors to $a_{1}$. When the excitation amplitude increases from a relatively small value, the unstable solutions increase sharply from zero. Soon after, the response gains stability at SN1 and decreases with the increase in excitation amplitude until SN2 occurs. At SN2, the response loses the stability and with the variation of excitation amplitude, the response increases 'inversely' and then decreases to zero sharply.
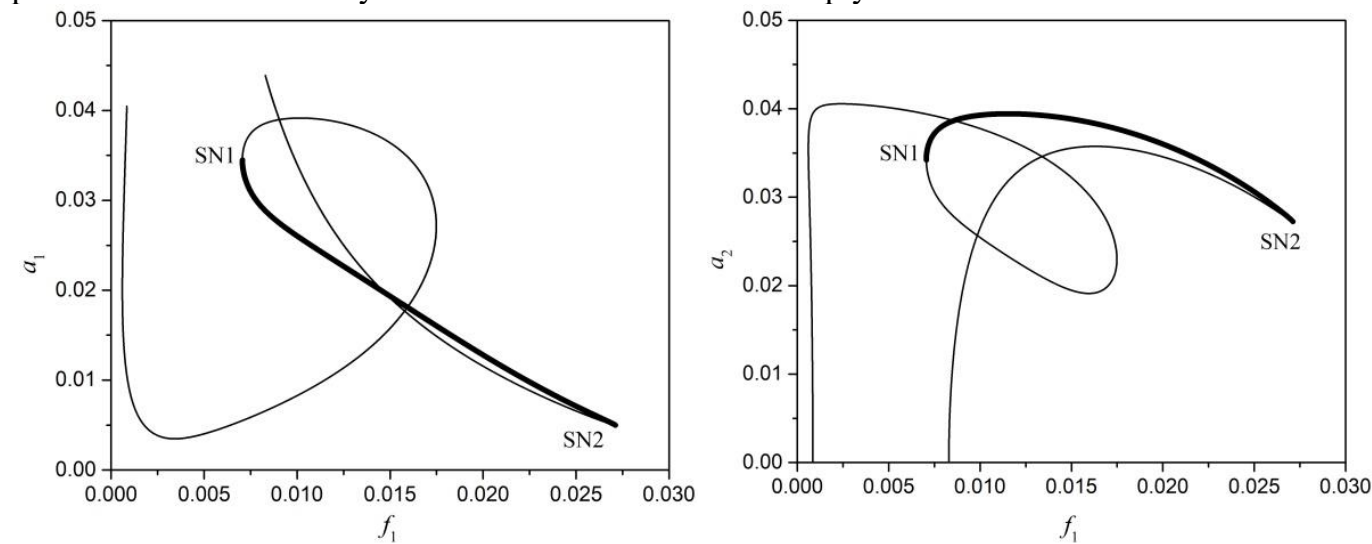

Fig. 4 The force-response curves of the CFRP cable when $\sigma=0.6$ and $\sigma_{1}=-0.0013$.

Fig. 5 illustrates the force-response curves of the CFRP cable with different external detuning parameters. It can be seen that with the increase in external detuning parameters, the range of the solutions becomes larger. All of the SN points gradually move to the upper right, which leads to that the range of stable solutions becomes larger. It seems that the whole curves are stretched. Moreover, the positions of the stable solutions gradually shift to the right with the increase in external detuning parameters, which means that the emergence of stable solutions is more difficult when external detuning parameter is larger. For example, if $f_{1}=0.01$, there are stable solutions when $\sigma=0.6$, while there are no stable solutions when $\sigma=0.75$ and $\sigma=0.9$. In other words, for a larger external detuning parameter (excitation frequency), the stable solutions may not be excited unless the excitation amplitude is large enough, which is very interesting. Actually, this can also be verified by the phenomena in Fig. 3 . 

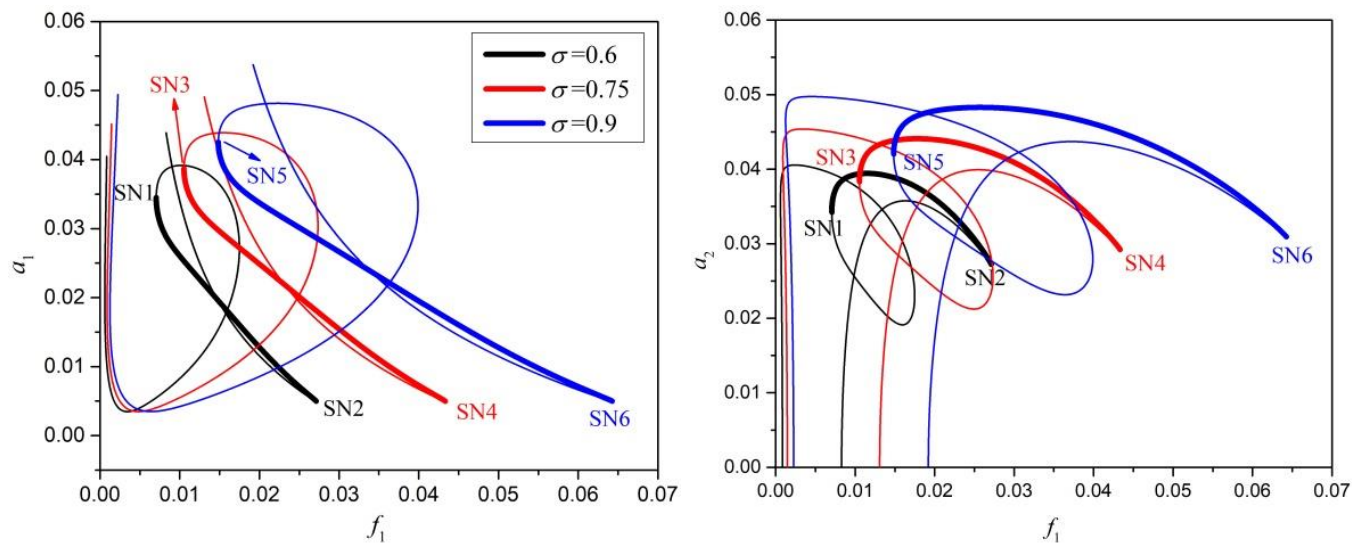

Fig. 5 The force-response curves of the CFRP cable with different external detuning parameters when $\sigma_{1}=-0.0013$.

Fig. 6 is the frequency-response curves of the CFRP cable and steel cable when excitation amplitude is 0.02 . It should be noted that the bifurcation points are not marked in Fig. 6, since they are not our focus. As can be seen in the figure, the nonlinear behaviors of the CFRP cable are simpler than those of the steel cable. The stable solutions of the CFRP cable are smaller than those of the steel cable. This indicates that the CFRP cable is a good choice to replace the steel cable. Similar phenomenon can also be seen in Fig. 7, which gives the force-response curves of the CFRP cable and steel cable when $\sigma=0.6$. As shown in the figure, the stable solutions of the CFRP cable are also smaller than those of the steel cable. However, the range of the stable solutions for the CFRP cable narrows, which is different from Fig. 6. In addition, when the steel cable is replaced by the CFRP cable, the stable solutions appear when the excitation amplitude is smaller, which is different with that of the steel cable.
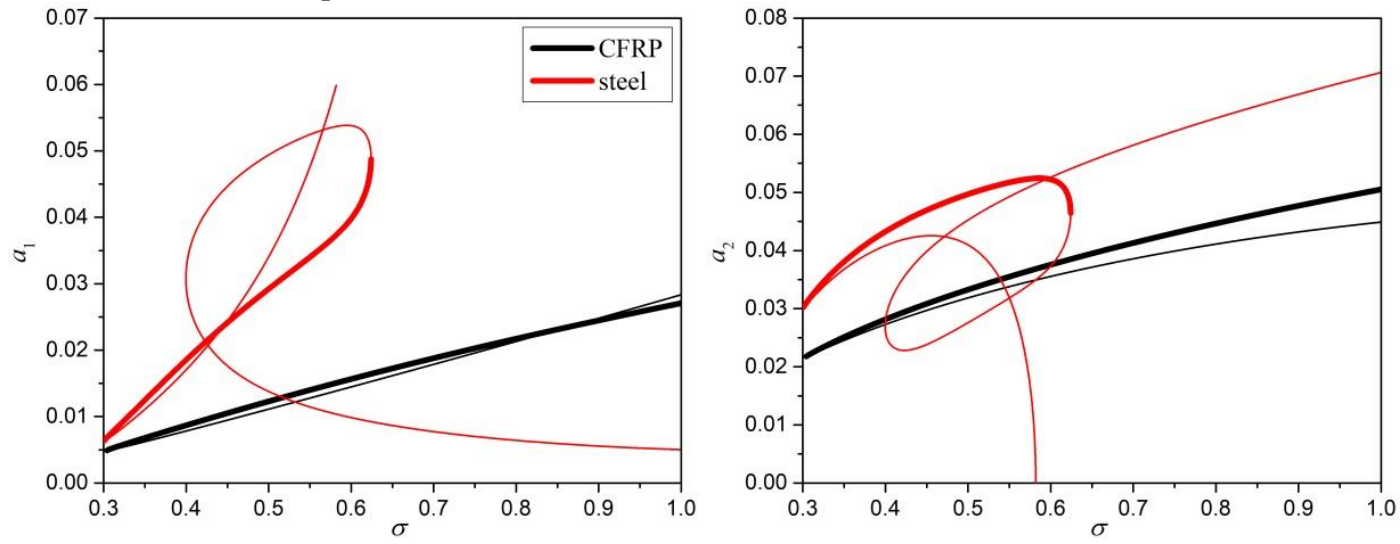

Fig. 6 The frequency-response curves of the CFRP cable and steel cable when excitation amplitude

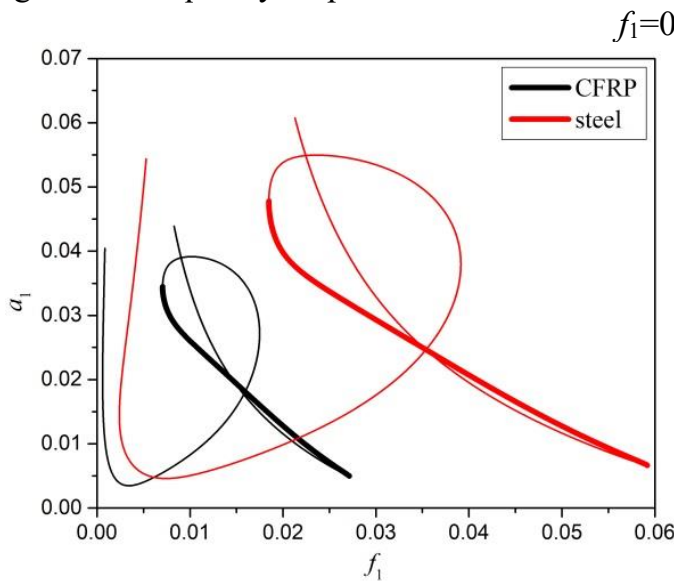
$f_{1}=0.02$.

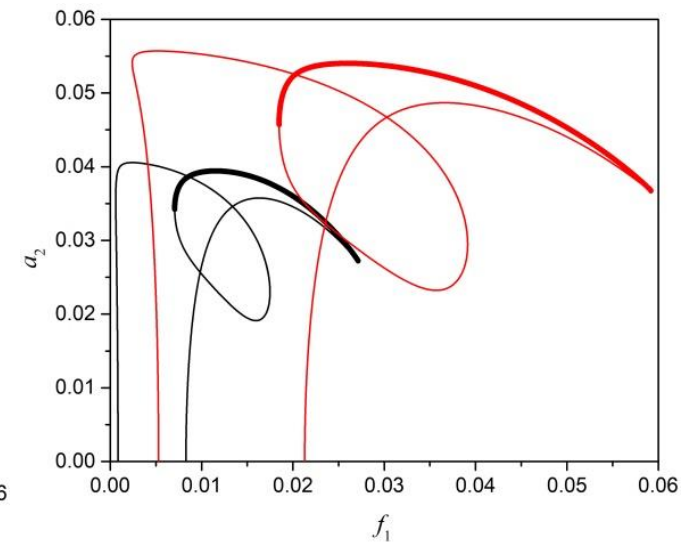

Fig. 7 The force-response curves of the CFRP cable and steel cable when $\sigma=0.6$.

\subsection{Out-of-plane excitation}

This part examines the nonlinear behaviors of the cable subjected to out-of-plane excitation, namely, 1/3-order subharmonic resonance of the out-of-plane mode. Fig. 8 presents the frequency-response 
curves of the CFRP cable with excitation amplitude $f_{1}=0.0035$. Compared with Fig. 2 , it seems that the nonlinear behaviors of out-of-plane motion are interchanged with those of in-plane motion. In other words, the response curve of $a_{1}$ in Fig. 8 is similar to that of $a_{2}$ in Fig. 2, while the response curve of $a_{2}$ in Fig. 8 is similar to that of $a_{1}$ in Fig. 2. In addition, $a_{1}$ is a little larger than $a_{2}$, which is opposite to the result in Fig. 2. It can be concluded that when the excitation is applied to in-plane (out-of-plane) mode, the response amplitude of out-of-plane (in-plane) vibration is a little bigger, which is very interesting.
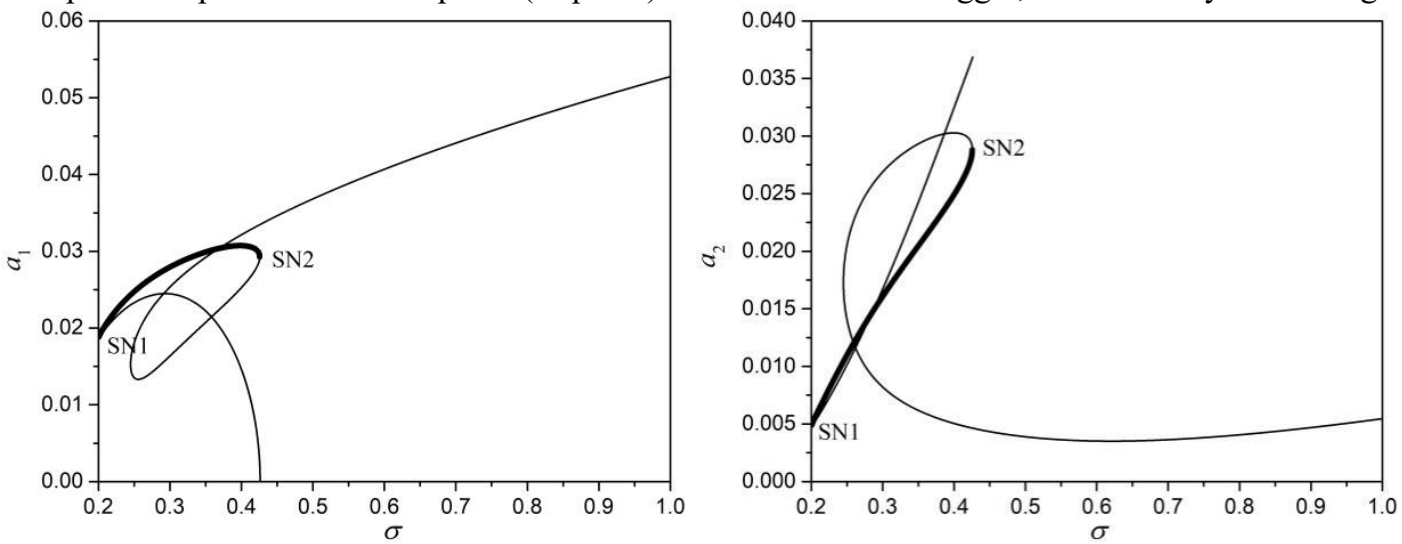

Fig. 8 The frequency-response curves of the CFRP cable with excitation amplitude $f_{1}=0.0035$ and $\sigma_{1}=-$ 0.0013 .

Fig. 9 shows the frequency-response curves of the CFRP cable with different excitation amplitudes. It can be seen from Fig. 9 that the shapes of the response curves hardly change because the variation of the excitation amplitude is small, which is different from Fig. 3. As the excitation amplitude is increased, the SNs gradually move to the upper right and the range of stable solutions becomes larger. In addition, the increase in excitation amplitude makes the frequencies corresponding to lower SNs (SN1, SN3 and SN5) become larger and it seems that the whole curves shift to the right. The above phenomenon indicates that when the excitation amplitude is greater, the required excitation frequency for the stable solutions is larger. Obviously, the excitation amplitude has a smaller effect on the response curves far away from the natural frequency ( $\sigma$ belongs to $[0.75,1.0])$.
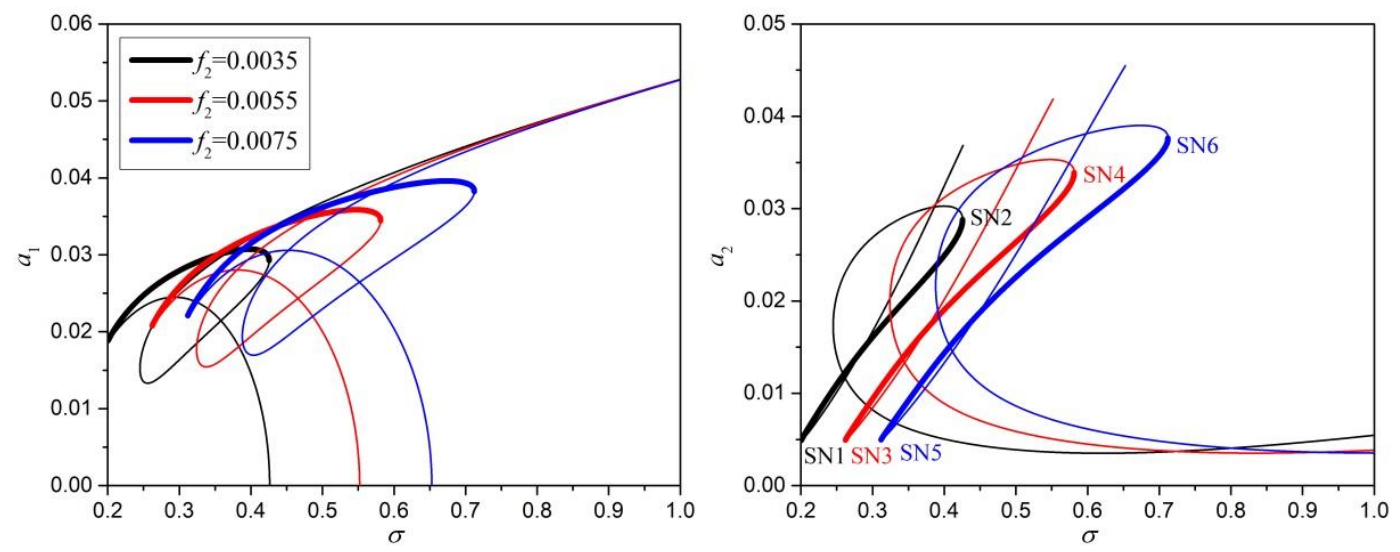

Fig. 9 The frequency-response curves of the CFRP cable with different excitation amplitudes when $\sigma_{1}=$ -0.0013 .

Fig. 10 presents the force-response curves of the CFRP cable when $\sigma=0.35$. Compared with Fig. 4 , the nonlinear behaviors of out-of-plane motion are also interchanged with those of in-plane motion. The difference is that there exist Hopf bifurcation points on the response curves in Fig. 10, which indicates that out-of-plane excitation makes nonlinear behaviors of the cable more complicated. It can be concluded from the above phenomena that there are significant differences between the nonlinear characteristics caused by the 1/3-order subharmonic resonance of different modes. To explore the effect of external detuning parameters on the nonlinear behaviors of the cable subjected to out-of-plane excitation, Fig. 11 presents the force-response curve of the CFRP cable with different external detuning parameters. As seen in the figure, the distance between two HBs (i.e. HB3 and HB4) is smaller with the increase in $\sigma$. However, the range of another stable branch expands. Although the response amplitudes increase with the increase in external detuning parameter, the resonance frequency range moves far away from the natural frequency. 

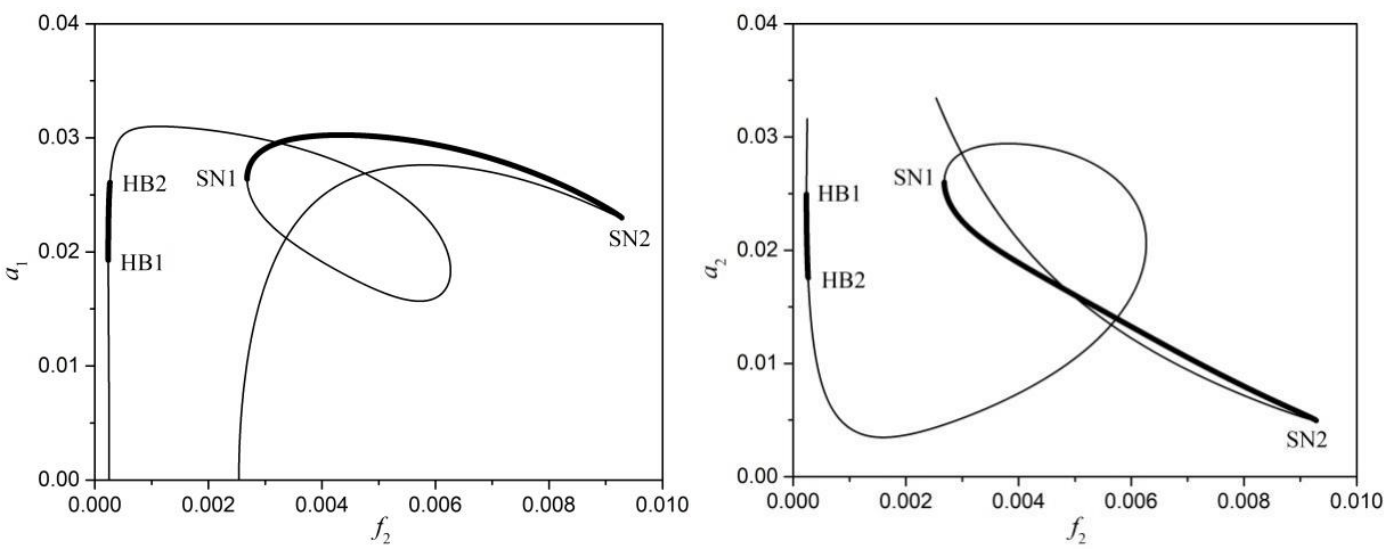

Fig. 10 The force-response curve of the CFRP cable when $\sigma=0.35$ and $\sigma_{1}=-0.0013$.
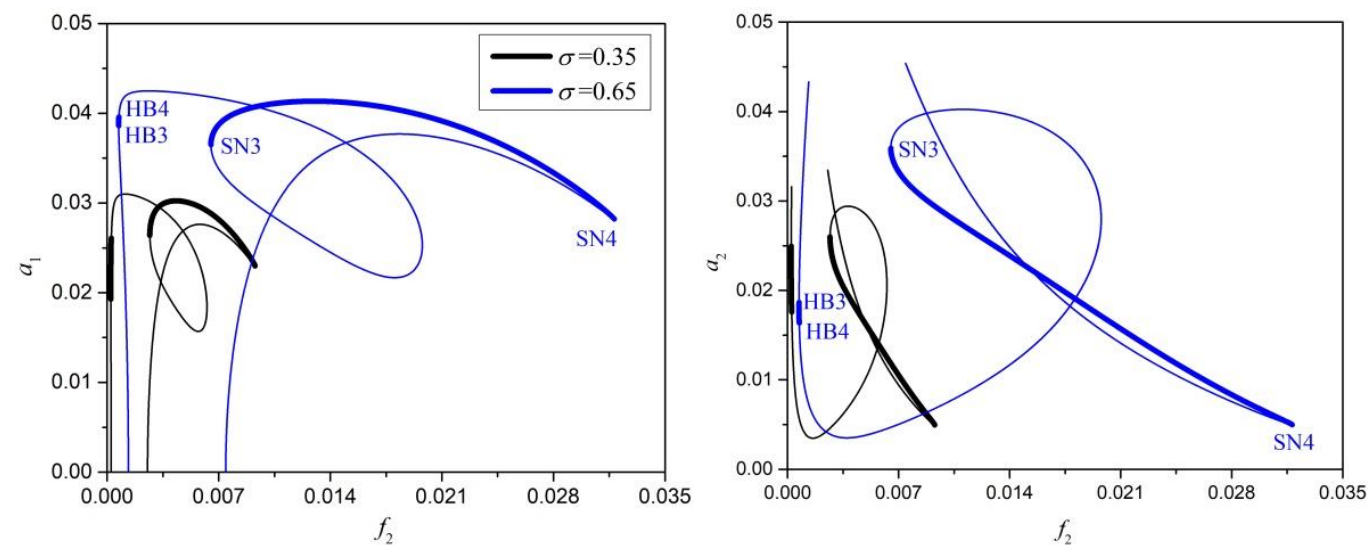

Fig. 11 The force-response curve of the CFRP cable with different external detuning parameters when $\sigma_{1}=-0.0013$.

4.3. Excitations in both of the in-plane and out-of-plane modes
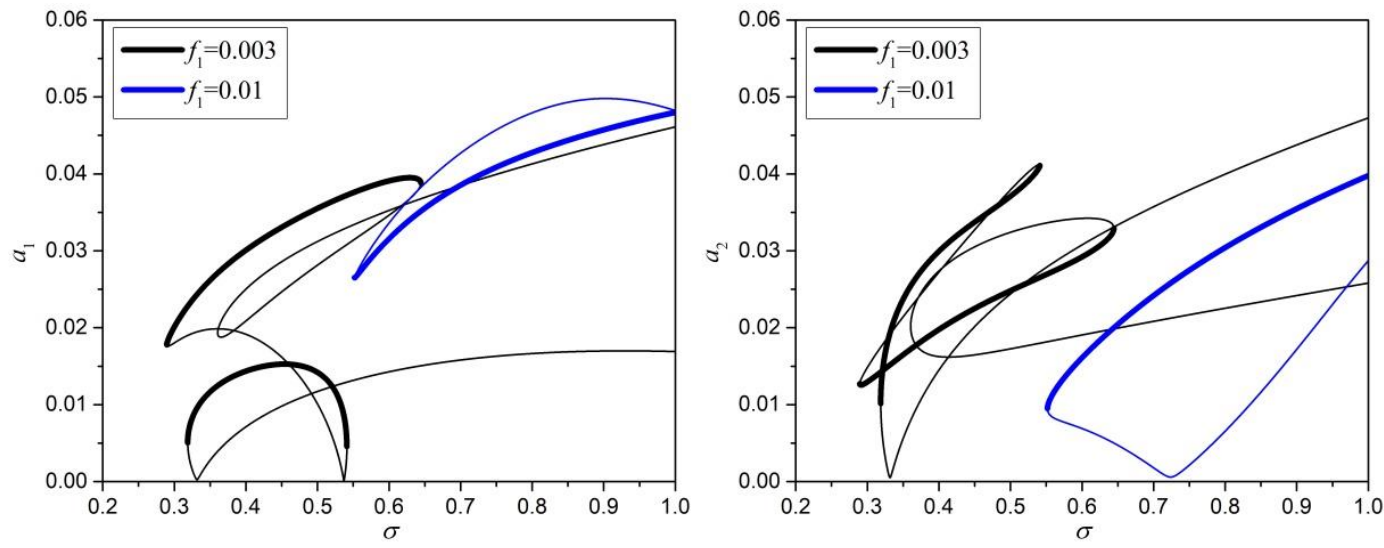

Fig. 12 The frequency-response curves of the CFRP cable with different excitation amplitudes when $\sigma=0.6, \sigma_{1}=-0.0013$ and $k=2$.

This part examines the nonlinear behaviors of the CFRP cable when external 1/3-order subharmonic resonances of both of the in-plane and out-of-plane modes occur. Fig. 12 gives the frequency-response curves of the CFRP cable with different excitation amplitudes. It should be noted that the bifurcation points are not marked in Fig. 12, since they are not our focus. There is no obvious softening or hardening characteristics in the response curves. As illustrated in the figure, the nonlinear behaviors of the cable are complex when the excitation amplitude is small. There are two stable branches when $f_{1}=0.003$, which means that there will be two stable solutions when $\sigma$ belongs to a certain frequency range. When the excitation amplitude is a little larger, the response curve corresponding to $a_{1}$ is a 
closed curve. In this case, the solutions only exist in a particular frequency region, which is just so-called frequency-island phenomenon [24,25]. Nevertheless, the response curve of $a_{2}$ is not closed when $f_{1}=0.01$, which indicates that the out-of-plane vibration may exhibit different nonlinear behaviors.

Fig. 13 gives the force-response curves of the CFRP cable with different external detuning parameters when $k=0.5$. The shapes of the force-response curves are clearly different from Fig. 4 and Fig. 10. As seen in Fig. 13, the nonlinear behaviors is relatively simple when the external detuning parameter is small (i.e. $\sigma=0.4$ ). There is only one stable branch and the range of stable solutions is small. With the increase in external detuning parameters, the nonlinear behaviors of the CFRP cable become more complicated and there are two stable branches in the response curves. Comparing Fig. 5, Fig. 11 with Fig. 13, it can be found that the variation trends of the response curves versus external detuning parameters are distinct for different excitation forms. In other words, with the variation of external detuning parameters, the shapes of response curves in Fig. 5 and Fig. 11 keep unchangeable. Only the amplitudes and positions of the response curves change. However, in Fig. 13, both of the shapes and amplitudes of the response curves change with the variation of external detuning parameters. This means that the nonlinear behaviors of the cable subjected to both of the in-plane and out-of-plane excitations are more complex and unordinary.
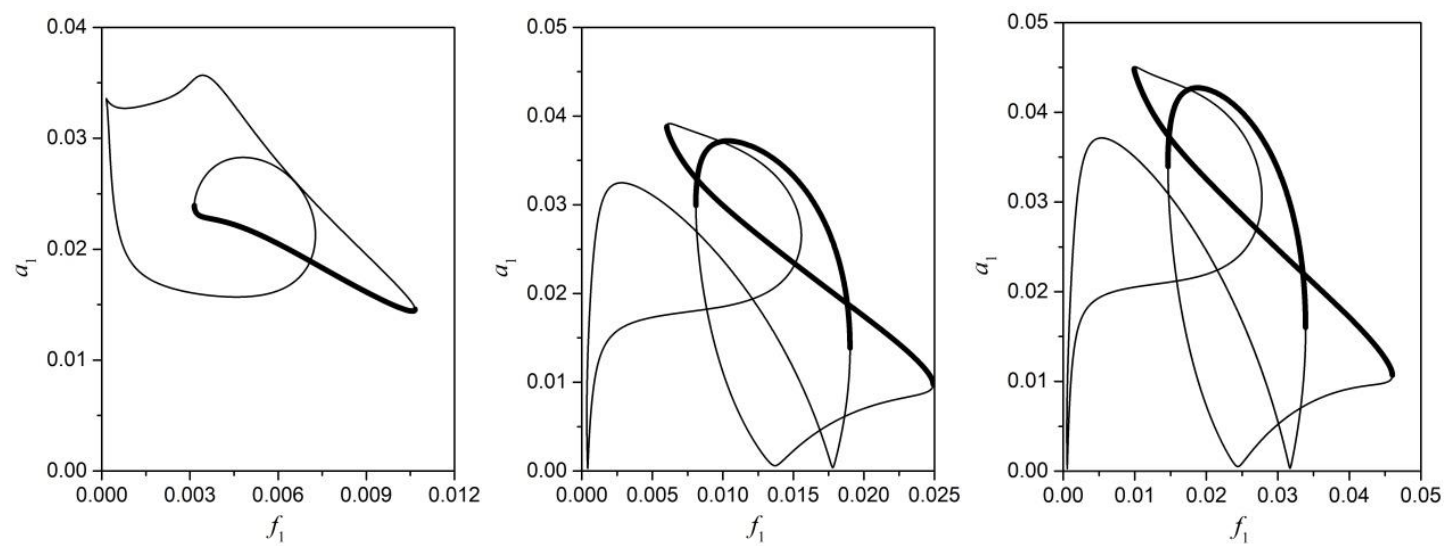

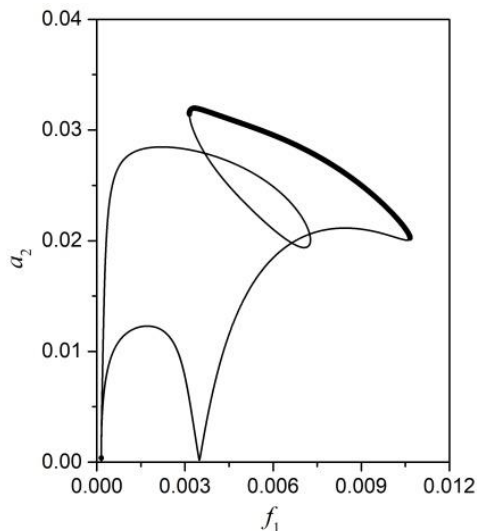

(a) $\sigma=0.4$

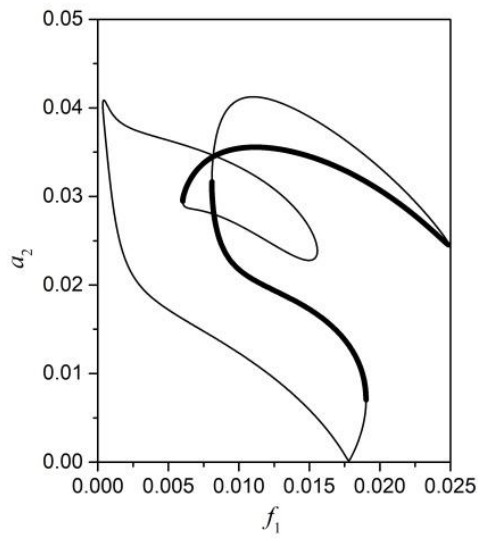

(b) $\sigma=0.6$

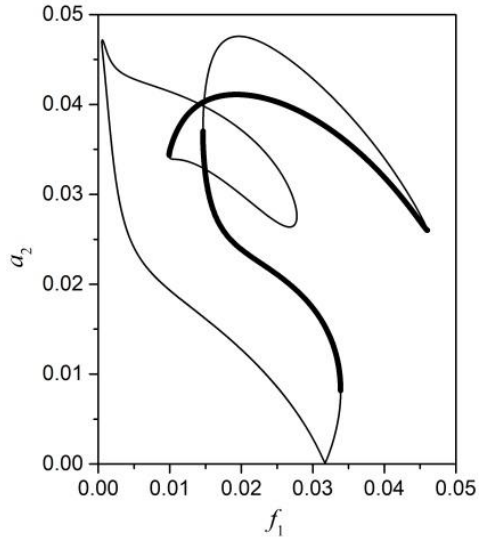

(c) $\sigma=0.8$

Fig. 13 The force-response curves of the CFRP cable with different $\sigma$ when $k=0.5$ and $\sigma_{1}=-0.0013$.

To explore the effect of the ratio of out-of-plane excitation amplitude to in-plane excitation amplitude on the nonlinear behaviors of the CFRP cable, Fig. 14 gives the force-response curves of the CFRP cable when $k$ is different. It can be seen that when $k=0.5$ and 2 , the nonlinear behaviors of the cable are relatively complex and there are two and three stable branches, respectively. However, there is only one stable branch when $k=1$. It can be concluded that when the in-plane and out-of-plane excitation amplitudes are different, the cable may exhibit more complicated behaviors. In addition, there are more stable solutions when the out-of-plane excitation is larger than in-plane excitation. When $\mathrm{k}=2$, the response amplitude will increase (decrease) sharply if the excitation is increased from zero. This is very dangerous in practical engineering, which should attract our attention. 

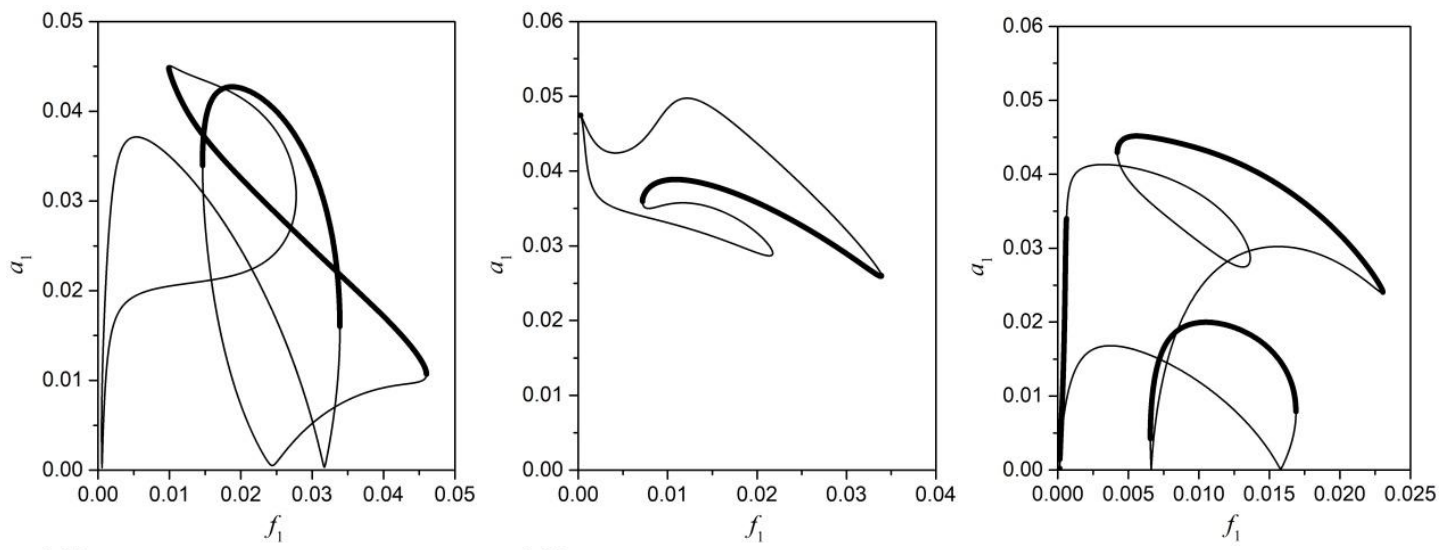

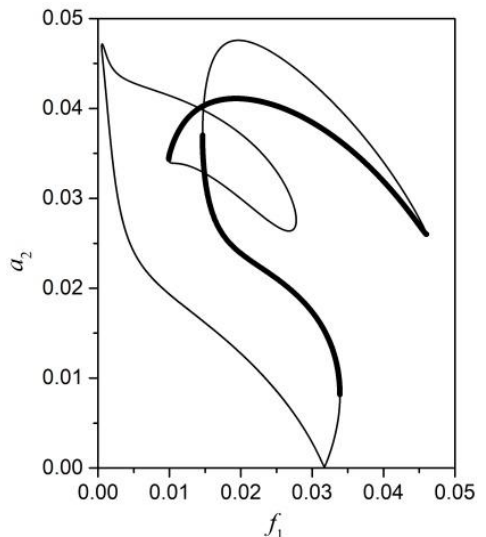

(a) $k=0.5$

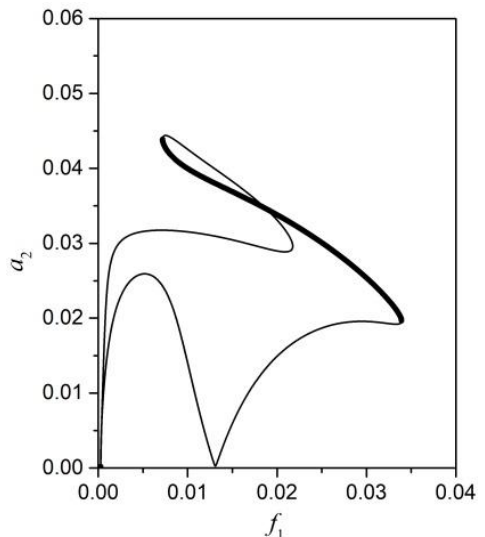

(b) $k=1$

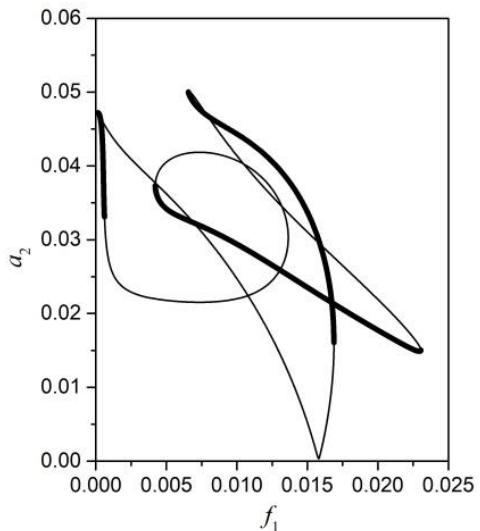

(c) $k=2$

Fig. 14 The force-response curves of the CFRP cable with $\sigma=0.8$ and $\sigma_{1}=-0.0013$ when $k$ is different.

Fig. 15 presents the comparison between force-response curves of the CFRP cable and steel cable when $k$ and $\sigma$ are different. As seen in the figure, the range of steady-state solutions of the CFRP cable is smaller than those of the steel cable, especially in Fig. 15 (c). When the steel cable is replaced by CFRP cable, the response amplitudes are greatly reduced. The solutions of both CFRP and steel cables start at $f_{1}=0$ and there is no difference at this point. However, the nonlinear behaviors of the steel cable are more complicated than those of the steel cable and there may be more bifurcation points on the response curves of the steel cable (see Fig. 15 (b) and (c)). The above phenomena again show that CFRP can improve the nonlinear behaviors of the cable and can be used to replace the steel cable from a nonlinear point of view. In addition, the nonlinear characteristics of the in-plane and out-of-plane motions are different, even though the excitation amplitudes of excitation are the same (i.e. $k=1$ ).
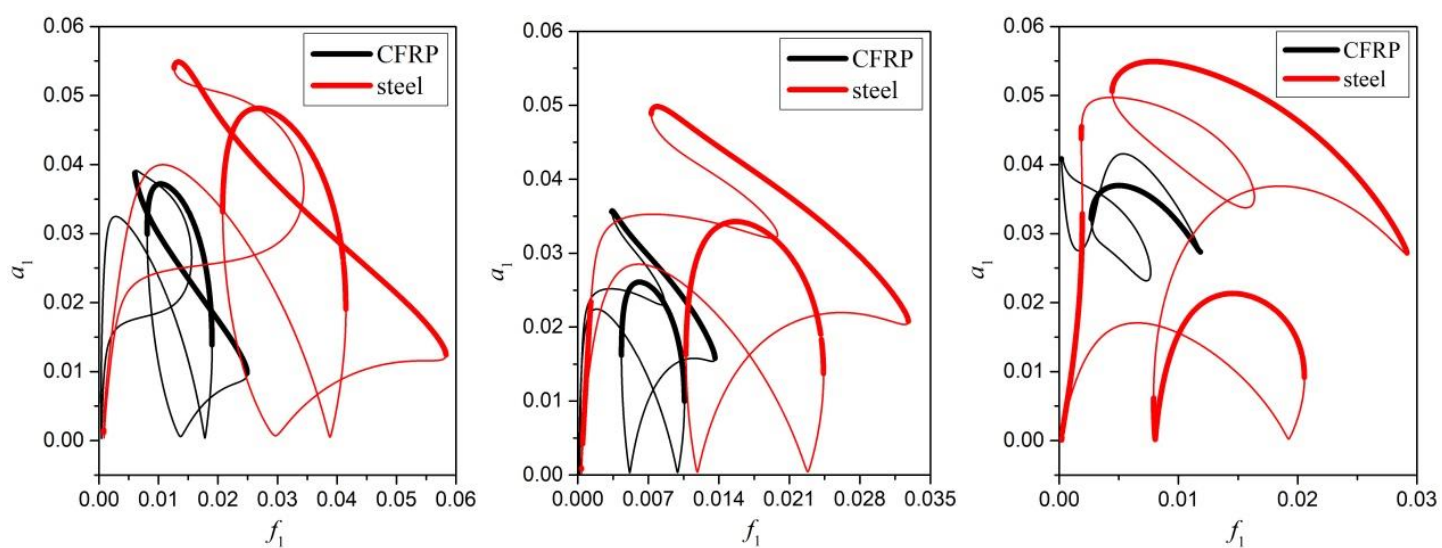


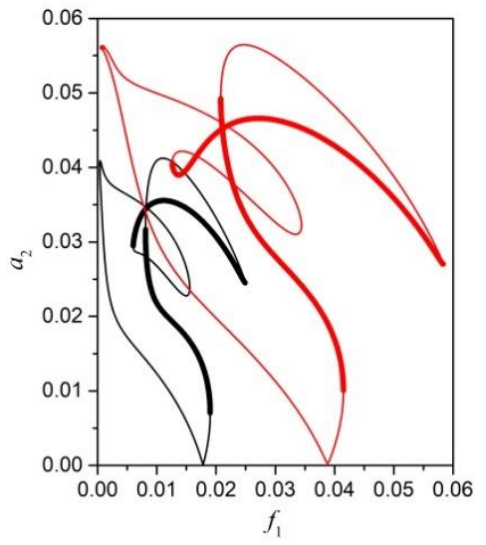

(a) $k=0.5$ and $\sigma=0.6$

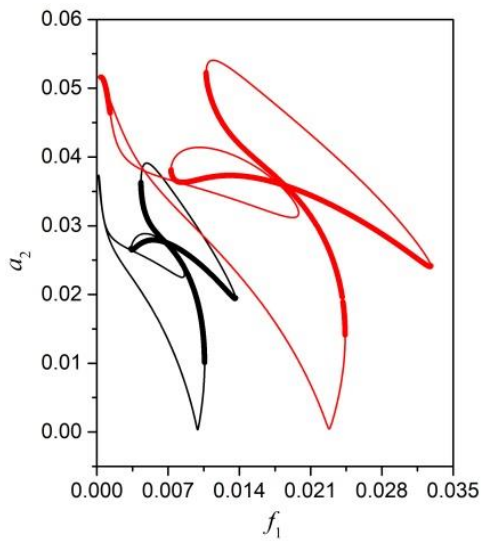

(b) $k=1$ and $\sigma=0.5$

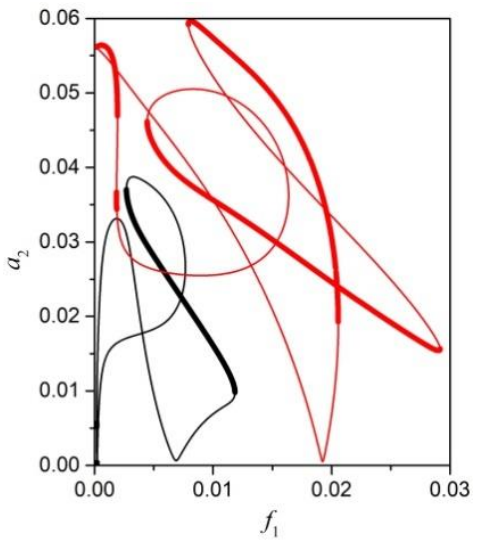

(c) $k=2$ and $\sigma=0.6$

Fig. 15 The force-response curves of the CFRP cable and steel cable with different $k$ and $\sigma$.

\section{Conclusions}

In order to explore the nonlinear behaviors of a CFRP cable when the external $1 / 3$ subharmonic resonance excited, the spatial motion of the cable is considered. Based on the vibration equations considering bending stiffness and shear rigidity, the one-to-one internal resonance between the lowest in-plane and out-of-plane modes is studied when external $1 / 3$-order subharmonic resonance occurs. Three different forms of excitations, namely, in-plane excitation, out-of-plane mode excitation and both of the in-plane and out-of-plane excitations are examined. Utilizing Galerkin's method, a series of ODEs are obtained and they are solved by multiple time scale method. After a standard solving process, the modulation equations of the system are derived. Then, one of the stable solutions of the modulation equations is acquired by Newton-Raphson method. Starting with the stable solution, the steady-state solutions can be continued by pseudo arclength algorithm. In this way, the response curves of the cable are drawn. Meanwhile, the response curves of the CFRP cable are compared with those of steel cable to reveal the superiorities of the CFRP cable. Finally, some conclusions are drawn as follows.

(1) The nonlinear behaviors of the CFRP cable will become simpler with the increase in excitation amplitude. The range of the solutions becomes larger with the increase in external detuning parameters. Under certain circumstances, energy transfer occurs through internal resonance. The in-plane motion exhibits almost the opposite nonlinear behaviors to out-of-plane motion.

(2) For a larger external detuning parameter (excitation frequency), the stable solutions may not be excited unless the excitation amplitude is large enough. The nonlinear behaviors under distinct forms of excitations are also different. The nonlinear behaviors of the cable subjected to both of the in-plane and out-of-plane excitations are more complex. There is no obvious softening or hardening characteristics in the response curves.

(3) Although the excitation amplitudes are the same $(k=1)$, the in-plane and out-of-plane motions may exhibit different nonlinear behaviors. The frequency-island phenomenon that the solutions only exist in a particular frequency region is observed. There are more stable solutions when the out-of-plane excitation is larger than in-plane excitation.

(4) On the whole, the response amplitude becomes smaller and the nonlinear behaviors are simpler when the steel cable is replaced by CFRP cable. From a nonlinear point of view, the performance of CFRP cable is better than that of steel cable, because the range of the large vibration is decreased.

\section{Acknowledgements}

The authors wish to acknowledge the support of the National Natural Science Foundation of China (11972151 and 11872176).

\section{Declarations}

Conflict of Interest: the authors declare that the have no conflict of interest.

Data Availability statement: Data sharing not applicable. 


\section{Appendix A}

For the sake of description, the following quantities are firstly introduced

$$
\begin{gathered}
B_{1}=\int_{0}^{1} y^{\prime}(x) \varphi^{\prime}(x) \mathrm{d} x ; \quad B_{2}=\frac{1}{2} \int_{0}^{1} \varphi^{\prime}(x) \varphi^{\prime}(x) \mathrm{d} x ; \quad B_{2}=\frac{1}{2} \int_{0}^{1} \phi^{\prime}(x) \phi^{\prime}(x) \mathrm{d} x ; \\
k_{1}=\int_{0}^{1}\left(\varphi(x) \varphi(x)-\xi \beta^{2} \varphi(x) \varphi^{\prime \prime}(x)\right) \mathrm{d} x ; \quad k_{2}=\int_{0}^{1}\left(\phi(x) \phi(x)-\xi \beta^{2} \phi(x) \phi^{\prime \prime}(x)\right) \mathrm{d} x
\end{gathered}
$$

In this way, the Galerkin integral coefficients in Eqs. (10) and (11) can be expressed as follows

$$
\begin{aligned}
& b_{11}=\frac{1}{k_{1}} \int_{0}^{1}\left(\beta^{2} \varphi(x) \varphi^{\prime \prime \prime}(x)-B_{1} \varphi(x) y^{\prime \prime}(x)-\frac{1}{\lambda} \varphi(x) \varphi^{\prime \prime}(x)+\frac{\xi \beta^{2}}{\lambda} \varphi(x) \varphi^{\prime \prime \prime}(x)\right) \mathrm{d} x \\
& b_{12}=\frac{1}{k_{1}} \int_{0}^{1}\left(B_{1} \xi \beta^{2} \varphi(x) \varphi^{\prime \prime \prime}(x)-B_{1} \varphi(x) \varphi^{\prime \prime}(x)-B_{2} \varphi(x) y^{\prime \prime}(x)\right) \mathrm{d} x \\
& b_{13}=\frac{1}{k_{1}} \int_{0}^{1} B_{3} \varphi(x) y^{\prime \prime}(x) \mathrm{d} x \\
& b_{14}=\frac{1}{k_{1}} \int_{0}^{1}\left(B_{2} \xi \beta^{2} \varphi(x) \varphi^{\prime \prime \prime}(x)-B_{2} \varphi(x) \varphi^{\prime \prime}(x)\right) \mathrm{d} x \\
& b_{15}=\frac{1}{k_{1}} \int_{0}^{1}\left(B_{3} \xi \beta^{2} \varphi(x) \varphi^{\prime \prime \prime}(x)-B_{3} \varphi(x) \varphi^{\prime \prime}(x)\right) \mathrm{d} x \\
& b_{21}=\frac{1}{k_{2}} \int_{0}^{1}\left(\beta^{2} \phi(x) \phi^{\prime \prime}(x)-\frac{1}{\lambda} \phi(x) \phi^{\prime \prime}(x)+\frac{\xi \beta^{2}}{\lambda} \phi(x) \phi^{\prime \prime \prime}(x)\right) \mathrm{d} x \\
& b_{22}=\frac{1}{k_{2}} \int_{0}^{1}\left(B_{1} \xi \beta^{2} \phi(x) \phi^{\prime \prime \prime}(x)-B_{1} \phi(x) \phi^{\prime \prime}(x)\right) \mathrm{d} x \\
& b_{23}=\frac{1}{k_{2}} \int_{0}^{1}\left(B_{2} \xi \beta^{2} \phi(x) \phi^{\prime \prime \prime}(x)-B_{2} \phi(x) \phi^{\prime \prime}(x)\right) \mathrm{d} x \\
& b_{24}=\frac{1}{k_{2}} \int_{0}^{1}\left(B_{3} \xi \beta^{2} \phi(x) \phi^{\prime \prime \prime}(x)-B_{3} \phi(x) \phi^{\prime \prime}(x)\right) \mathrm{d} x
\end{aligned}
$$

\section{References}

[1] Su, X.Y., Kang, H.J., Chen, J.F., Guo, T.D., Sun, C.S., Zhao, Y.Y.: Experimental study on in-plane nonlinear vibrations of the cable-stayed bridge. Nonlinear Dyn. 98(2), 1247-1266 (2019)

[2] Xu, L., Hui, Y., Yang, Q.S., Chen, Z.Q., Law, S.S.: Modeling and modal analysis of suspension bridge based on continual formula method. Mech. Syst. Sig. Process. 162, 107855 (2022)

[3] Irvine, H.M.: Cable Structures. Dover Publications, New York (1992)

[4] Hagedorn P, Schäfer B. On non-linear free vibrations of an elastic cable. International Journal of Non-Linear Mechanics, 1980, 15(4-5): 333-340

[5] Luongo A, Rega G, Vestroni F. Planar non-linear free vibrations of an elastic cable. International Journal of Non-Linear Mechanics, 1984, 19(1): 39-52

[6] Benedettini F, Rega G. Non-linear dynamics of an elastic cable under planar excitation. International Journal of Non-Linear Mechanics, 1987, 22(6): 497-509

[7] Benedettini, F., Rega, G.: Planar non-linear oscillations of elastic cables under superharmonic resonance conditions. J. Sound Vib. 132(3), 353-366 (1989)

[8] Rega, G., Benedettini, F.: Planar non-linear oscillations of elastic cables under subharmonic resonance conditions. J. Sound Vib. 132(3), 367-381 (1989)

[9] Perkins N C. Modal interactions in the non-linear response of elastic cables under parametric/external excitation. International Journal of Non-Linear Mechanics, 1992, 27(2): 233250

[10] Gattulli V, Vestroni F. Nonlinear strategies for longitudinal control in the stabilization of an oscillating suspended cable. Dynamics and Control, 2000, 10(4): 359-374

[11] Zhao, Y.Y., Wang, L.H.: On the symmetric modal interaction of the suspended cable: three-to-one internal resonance. J. Sound Vib. 294(4-5), 1073-1093 (2006)

[12] Wang L H, Zhao Y Y. Nonlinear interactions and chaotic dynamics of suspended cables with three-to-one internal resonances. International Journal of Solids and Structures, 2006, 43(25-26): 
7800-7819

[13] W. Lacarbonara, G. Rega, Resonate nonlinear normal modes. Part II: activation/orthogonality conditions for shallow structural systems, International Journal of Non-linear Mechanics 38 (2003) 873-887

[14] Wang L H, Zhao Y Y. Multiple internal resonances and non-planar dynamics of shallow suspended cables to the harmonic excitations. Journal of Sound and Vibration, 2009, 319(1-2):114.

[15] XIE, X., LI, X Z., and SHEN, Y G. Static and dynamic characteristics of a long-span cable-stayed bridge with CFRP cables. Materials, 7, 4854-4877 (2014)

[16] Ren L, Fang Z, Wang K. Design and behavior of super-long span cable-stayed bridge with CFRP cables and UHPC members. Composites Part B: Engineering, 2019, 164: 72-81.

[17] Su, X.Y., Kang, H.J., Guo, T.D., Cong, Y.Y.: Modeling and parametric analysis of in-plane free vibration of a floating cable-stayed bridge with transfer matrix method. Int. J. Struct. Stab. Dyn. 20, $2050004(2020)$

[18] MEI, K H., SUN, S J., JIN, G P., and SUN, Y M. Static and dynamic mechanical properties of long-span cable-stayed bridges using CFRP cables. Advances in Civil Engineering, 2017, 1-11 (2017)

[19] Xie G H, Yin J , Liu R G, Chen B, Cai D S. Experimental and numerical investigation on the static and dynamic behaviors of cable-stayed bridges with CFRP cables. Composites Part B Engineering, 111(2016):235-242.

[20] Kang H J, Zhu H P, Zhao Y Y, Yi Z P. In-plane non-linear dynamics of the stay cables. Nonlinear Dynamics. 2013, 73(3): 1385-1398.

[21] Kang H J, Guo T D, Zhu W D, Su J Y, Zhao B Y. Dynamical modeling and non-planar coupled behavior of inclined CFRP cables under simultaneous internal and external resonances. Applied Mathematics and Mechanics (English Edition), 40(5), 649-678 (2019)

[22] Seydel, R.: Practical bifurcation and stability analysis. Springer, New York. (2009).

[23] Nayfeh, A.H., Balachandran, B.: Applied nonlinear dynamics. Wiley, New York. (1995).

[24] Rega G, Srinil N. Nonlinear hybrid-mode resonant forced oscillations of sagged inclined cables at avoidances. J. Comput. Nonlin. Dyn. 2007, 2(4):324-336.

[25] Lacarbonara, W., Arafat, H.N., Nayfeh, A.H.: Non-linear interactions in imperfect beams at veering. Int. J. Nonlinear Mech. 40, 9871003 (2005) 